\title{
HUMAN MEIOSIS VI. CROSSING OVER IN HUMAN SPERMATOCYTES
}

\author{
by \\ PREBEN BACH HOLM \\ and \\ SØREN WILKEN RASMUSSEN \\ Department of Physiology, Carlsberg Laboratory \\ Gamle Carlsberg Vej 10, DK-2500 Copenhagen Valby
}

Keywords: Synaptonemal complex, recombination nodule, bar, gene conversion, non-disjunction, terminalization, interference.

The structure, number and distribution of recombination nodules in human spermatocytes have been analyzed by serial sectioning and three dimensional reconstruction of 10 late zygotene and 73 pachytene nuclei, the pachytene nuclei being allocated to seven substages. The analysis has permitted the following observations and conclusions. 1) Recombination nodules bind to the central region of the synaptonemal complex at zygotene, increase in size and transform, at pachytene stage 2, into a bridge-like structure termed a bar. During pachytene stages 3 and 4 , the bars become more spindle shaped and are often oriented obliquely to the synaptonemal complex. By pachytene stages $5-7$ the bars appear to resolve into filaments and disappear eventually. 2) The number of nodules decreases from a mean of 101 at late zygotene ( 72 percent pairing) to 75 at pachytene stage 1 . At pachytene stage 2, 58 nodules and 16 bars are present, the corresponding figures for pachytene stages 3,4 and 5 being 11 and 35, 6 and 35 and 7 and 24 . At the later stages very few nodules and bars are seen. 3) Nodules are distributed at random among the bivalents and the bivalent arms at zygotene while at pachytene stage $3+4,99$ percent of the bivalents and 75 percent of the bivalent arms have at least one nodule or bar. By stage 5 the nodulebar distribution is to a large extent random. 4) The structural, numerical and distributional changes of nodules and bars suggest that the transformation of a nodule into a bar reflects a crossing over event. 5) As not all crossovers of a nucleus can be observed at the same time, the total crossover distribution was constructed by cumulating the frequencies of bars at stage 2 and the nodules and bars at stage 3-7. The frequency of crossing over is high in the distal segments and low near the centromeres. In longer bivalent arms crossing over is also frequent in one or more interstitial segments. Crossing over occurs in 75 percent of the $\mathrm{XY}$ bivalents as judged by the frequency of nodules in the synaptonemal complex which combines the homologous regions of the $X$ and $Y$ chromosomes. 6) The total number of crossovers is estimated to range from 69 to 73 per nucleus and compares with a mean of 50 chiasmata at diakinesis. 7) The distribution of chiasmata at diakinesis and that of crossovers at pachytene differs, especially in the short arms. 8) One crossover (rarely two) in the form of a recombination structure is present in the short bivalent arms. Several crossovers are present in the longer arms, but preferentially placed in the middle of two or three domains. Once a crossover has occurred in a domain, the probability for a second crossover to be realized in the same domain is decreased. The limitation to the placement of a single crossover in a short arm and in a domain of a long arm is a source for positive interference in genetic linkage analyses. 


\section{INTRODUCTION}

A considerable number of investigations have supported the proposal by CARPENTER (9) that small electron dense nodes - recombination nodules - associated with the synaptonemal complex are involved in meiotic recombination: (1) The number and distribution of nodules are similar to the number and distribution of crossovers and/or chiasmata $(7,9,10,19,25,28,37,50)$. (2) Females of the silkworm, Bombyx mori, in which crossing over does not take place, also lack recombination nodules (44). (3) Meiotic mutants of Drosophila melanogaster, exhibiting a reduced frequency and altered distribution of crossing over, show corresponding changes in the number and distribution of recombination nodules (11). (4) Reconstructed nuclei from the male silkworm and the basidiomycete Coprinus cinereus covering the period from zygotene to metaphase I document the morphological conversion of recombination nodules into distinct chiasmata $(25,26)$. (5) Bivalent regions containing recombination nodules show increased DNA repair activity at pachytene as measured by 3H-thymidine autoradiography of electron micrographs (12).

Recombination nodules are prerequisites for, not the result of a recombination event as documented by the presence of recombination nodules in central region material accumulating in the nucleolus of Neottiella (49) at leptotenezygotene or by their association with only one of the lateral components in triploid Coprinus meiocytes (45). Nodules are also present in nonhomologously paired chromosome segments in Triticum (21), and in the recombination deficient mutant mei- 9 of Drosophila. In the latter, the number of nodules is similar to that seen in the wild type despite a drastic decrease in recombination frequency (11). Hence, the presence of a nodule reflects a potential for recombination which may or may not be exploited.

Recombination nodules associate with the synaptonemal complex already at zygotene. The initial binding of nodules to the synaptonemal complex occurs at random among the bivalents $(25,26,42)$ and the characteristic nonrandom distribution of recombination nodules, which matches that of crossovers is first achieved at mid-late pachytene following numerical and dis- tributional changes of the nodules during zygotene and early pachytene $(25,26,42)$. These changes are accompanied by an increase in size and density of the nodule.

The purpose of the present paper is to extend the previously published information on recombination nodules in the human male (42), to elucidate the mechanisms which regulate the frequency as well as the distribution of crossing over. As extensive data are available on the number and localization of chiasmata in the human male $(29,34)$, the relationship between recombination nodules ( $=$ crossovers) and chiasmata can be studied and the notion that chiasmata at diakinesis are a true measure of crossing over frequency and distribution tested.

\section{MATERIALS AND METHODS}

\subsection{Materials}

The material consists of 83 completely reconstructed nuclei and is from the same biopsies (cases 1-5 and a translocation heterozygote $t(5 ; 22))$ as those used previously $(23,24,27,42$, 43). Case 1 is an azoospermic man with apparently normal spermatogenesis and cases $2-5$ are normal fertile men. As no differences with respect to the structure, number and distribution of recombination nodules were observed among the six cases, the data have been pooled. The ten late zygotene nuclei and the twenty-one early pachytene nuclei (stage 1 of pachytene, see section 3.1.) have been described previously (42).

\subsection{Methods}

The procedures used for fixation, staining, electron microscopy and the subsequent reconstruction of the chromosome complement by its synaptonemal complexes as well as the computerized methods for measuring the synaptonemal complex length have been described previously $(23,42)$.

\section{RESULTS}

\subsection{Substages of pachytene}

The analysis of 73 completely reconstructed nuclei covering the interval from late zygotene to early diplotene revealed a continuous sequence of changes in fine structure and morphology which permitted a subdivision of this period into seven substages. As described in the preceding 
paper of this series (27), each substage was characterized by a combination of morphological features relating to (1) the autosomal chromatin, (2) the centromere region, (3) the XY bivalent, (4) the secondary constriction on bivalents 1, 9 and $16,(5)$ the nucleolus and $(6)$ the centrioles.

\subsection{Recombination nodules and bars}

Recombination nodules attach to the central region of the synaptonemal complex at early zygotene and continue to be incorporated throughout zygotene, either at the pairing fork or by binding to fully organized synaptonemal complexes (42). The nodules at zygotene are small and spherical with a minimum diameter of $30 \mathrm{~nm}$ or less frequently, shaped like a rod or a dumbbell.

From late zygotene to pachytene stage 1 , the number of nodules decreases from a mean of 101 to 75 per nucleus ( 42 , and Table I). The remaining nodules increase in size and density and faint connections are often observable between the nodules and the lateral components. At pachytene stage 2 , the nodules attain their maximum size, the mean number per nucleus being 73 ,i.e., almost the same as at stage 1. Several nodules have changed shape (Figures la and b), consisting of a more prominent bar lying at a right angle to the lateral components and bridging the central region, and a less distinct part, resembling the early small nodule, oriented in parallel with and lying on the central region. In some cases (mean of 16 per nucleus, Table I), the latter part of the nodule cannot be recognized (Figures 1c and d). Such nodules are referred to as bars while the term "nodule" includes intermediates between nodules and bars. Although recombination nodules at zygotene and pachytene stage 1 occasionally appear to be connected to the lateral components by fine fibrillar connections, nodules consisting exclusively of a prominent bar across the central region are rarely observed at pachytene stage 1 and never at late zygotene.

By stage 3 (Figures 2a-d), the nuclei on the average contain 35 bars and 11 nodules. Hence, a sixfold reduction in the total number of nodules (from 58 to 11) has taken place between stages 2 and 3 accompanied by a doubling of the number of bars (Table I). Several bars deviate structurally from those at stage 2 by having a more even and fusiform outline (Figures 3a-d) and while the bars at stage 2 are always oriented at a right angle to the lateral components, the fusiform bars are often located obliquely to the complex. Such bars are the most abundant type also at stage 4 , their mean number being equal to that found at stage 3 whereas the number of nodules has decreased further (Table I). Several of the bars observed at stage 4 are less dense and a few appear to have resolved into tufts of filaments (Figures 4a-d), often without contact to one or both lateral components. In those cases where the spindle shape of the bar is partially or entirely lost, the distinction between nodules and bars is ambiguous and it is conceivable that some structures

Table I.

Total complement length, number of recombination nodules and number of recombination bars in human spermatocytes. (sd, standard deviation).

\begin{tabular}{lccccc}
\hline Stage & $\mathrm{n}$ & $\begin{array}{c}\text { Complement length } \\
(\mu \mathrm{m} \pm \mathrm{sd})\end{array}$ & $\begin{array}{c}\text { Number of } \\
\text { recombination } \\
\text { nodules } \\
( \pm \mathrm{sd})\end{array}$ & $\begin{array}{c}\text { Number of } \\
\text { recombination } \\
\text { bars } \\
( \pm \mathrm{sd})\end{array}$ & $\begin{array}{c}\text { Total number } \\
\text { of recomb. } \\
\text { structures } \\
( \pm \mathrm{sd})\end{array}$ \\
\hline Late zygotene & 10 & $235 \pm 26$ & $101 \pm 25$ & 0 & $101 \pm 25$ \\
Pachytene 1 & 21 & $207 \pm 23$ & $75 \pm 20$ & 0 & $75 \pm 20$ \\
Pachytene 2 & 12 & $215 \pm 16$ & $58 \pm 14$ & $16 \pm 7$ & $73 \pm 14$ \\
Pachytene 3 & 15 & $235 \pm 13$ & $11 \pm 5$ & $35 \pm 6$ & $45 \pm 6$ \\
Pachytene 4 & 5 & $239 \pm 17$ & $6 \pm 5$ & $35 \pm 2$ & $42 \pm 6$ \\
Pachytene 5 & 9 & $223 \pm 9$ & $7 \pm 4$ & $24 \pm 8$ & $31 \pm 7$ \\
Pachytene 6 & 4 & $239 \pm 18$ & $6 \pm 2$ & $12 \pm 9$ & $18 \pm 9$ \\
Pachytene 7 & 7 & $198 \pm 16$ & $1 \pm 1$ & $4 \pm 2$ & $5 \pm 2$ \\
\hline
\end{tabular}



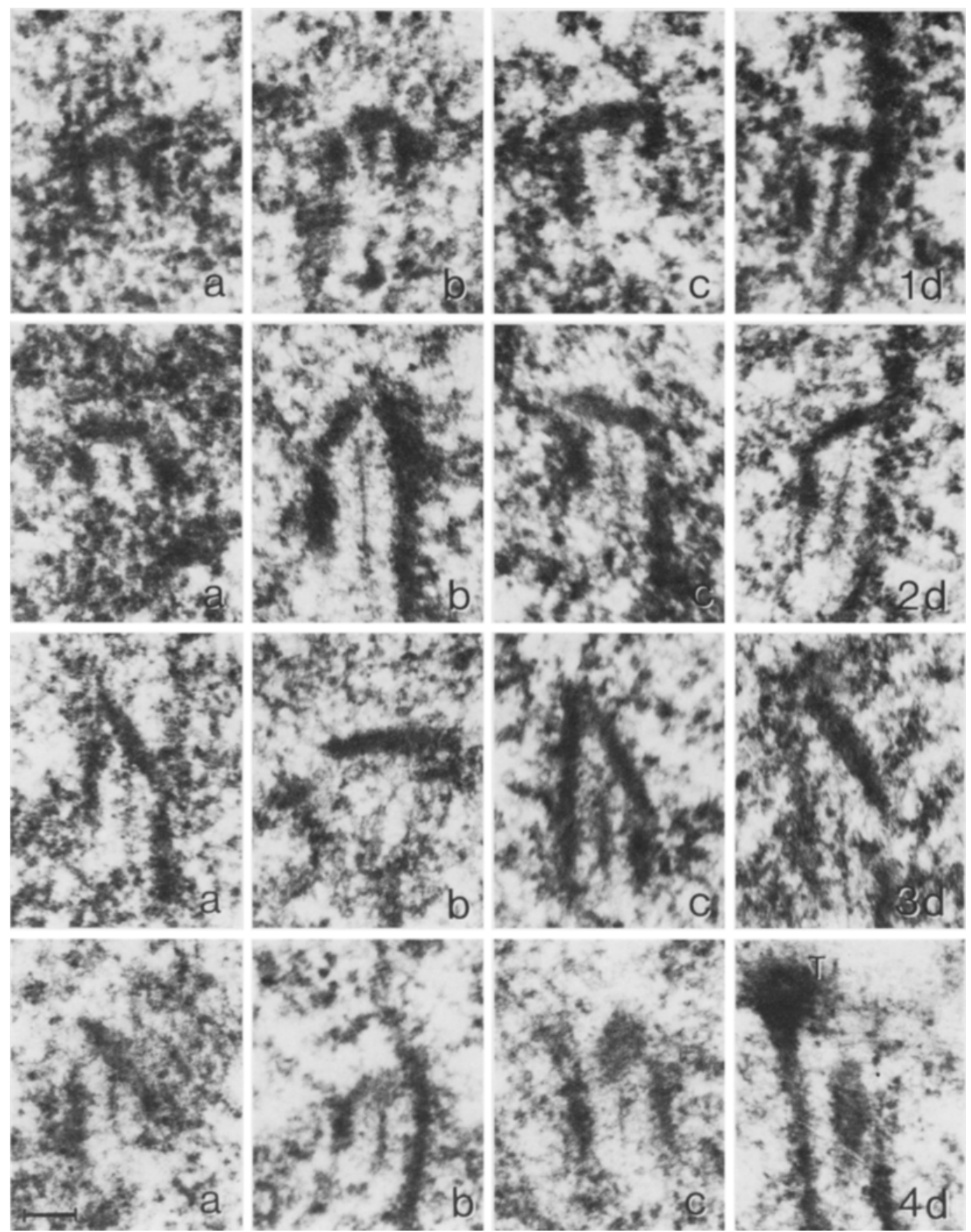

Figures 1-4. Electron micrographs showing the transformation of " $T$ " shaped recombination nodules into bars at stage 2 (Figures la-d), bars at stage 3 (Figures $2 a-d$ ), spindle shaped bars lying at an oblique angle on the synaptonemal complex at stages 4 and 5 (Figures 3a-d) and resolving bars at stages 4 and 5 (Figures $4 a-d)$. T, telomere. $($ Bar $=$ $0.1 \mu \mathrm{m})$. 
classified as nodules at this as well as the following stages rather represent resolving bars. As can be seen in Table I, the number of bars decreases between stages 4 and 5 from a mean of 35 to 24 . At stage 6 , a further reduction occurs and by stage 7 , the last substage of pachytene, virtually all nodules and bars have disappeared.

\subsection{The $X Y$ bivalent}

In all nuclei analyzed at pachytene stage 1 , the short arm of the $Y$ chromosome and the distal segment of the short arm of the $\mathrm{X}$ chromosome are paired and have a synaptonemal complex or are in the process of acquiring one. The XY bivalent lacks a recombination nodule in six nuclei ( 27 percent) while the remaining sixteen possess one or more nodules. At pachytene stage 2, all $12 \mathrm{XY}$ bivalents analyzed possess a short synaptonemal complex segment of which nine contain one or more nodules or intermediates between nodules and bars.

Synaptonemal complexes in XY bivalents are rarely seen at stages 3-6, apparently as a result of extensive structural modification of the lateral components, and in the cases where synaptonemal complex segments were identified, recombination nodules and bars were never seen. This is also the case at stage 7, where the structure of the $X Y$ bivalent reverts towards that at pachytene stages 1 and 2 (see 27 for a description of the morphological changes of the $\mathrm{XY}$ bivalent). The observation that only intermediates between nodules and bars are seen in the $X Y$ bivalent of human spermatocytes may somehow be related to the temporal disappearance of the synaptonemal complex segment in the $X Y$ bivalent concomitant with the appearance of bars in the complexes of the autosomal bivalents.

\subsection{Distribution of nodules and bars among bivalents and bivalent arms}

The observed distributions of nodules and bars among the bivalents and the bivalent arms at late zygotene and at pachytene stages 1, 2, 3+ 4 and 5 were compared with the distributions expected if nodules and bars were distributed at random among and along the bivalents in order to assess to what extent the changes of the total number of nodules and bars as well as the ratio between nodules and bars, are accompanied by changes of the way these structures are distributed. The random distributions were obtained by a computer simulation procedure as follows: The absolute length and the centromere indices of the autosomal bivalents of each nucleus, as well as the total number of nodules and bars of the individual nuclei, were fed into a Honeywell Bull $\mathrm{H} 6000$ computer programmed to position the nodules/bars at random on the bivalent complement. In partially paired zygotene bivalents, the unpaired regions were excluded by joining the paired segments. The nodules/bars were positioned one at a time and, in those cases where the position generated by the computer was closer than $80 \mathrm{~nm}$ to a previously positioned nodule or closer than $40 \mathrm{~nm}$ to a telomere or a centromere, the position was rejected and a new one generated. The program was run a total of 10,800 times for each stage, the number of runs for a given nucleus corresponding to 10,800 divided by the number of nuclei reconstructed for that stage. In this way, the frequencies of bivalents and bivalent arms with 0,1 , $2, \ldots>7$ nodules/bars were obtained as well as the relative distribution of recombination structures between the arms of bivalents with 2, 3 and 4 nodules/bars.

The distribution of nodules among bivalents and bivalent arms at late zygotene appears to be at random as judged by the virtual identity between the frequencies of bivalents with 0,1 , $2, \ldots>7$ nodules and those obtained after a random positioning of the nodules ( Tables II and III). The nodules and bars at pachytene stages 1 and 2 are also distributed among the bivalents nearly at random with slightly more bivalents containing one or two nodules/bars than can be accounted for by a random positioning. A marked deviation from randomness is, however, apparent between the frequencies of bivalents without nodules/bars, where at stage 1 only about half and at stage 2 one-seventh of the expected frequency following a random placement are actually observed (Table II). The deviation from randomness in the placement of nodules/bars culminates at pachytene stages 3 and 4 , the observations from the two stages being pooled as the number of nodules and bars at the two stages were virtually identical (Table I). Only about one 
Table II.

Distribution of recombination nodules, bars and chiasmata among the bivalents of human spermatocytes. $O$, observed frequencies. $E$, expected frequencies if nodules and bars are distributed randomly among the bivalents.

\begin{tabular}{|c|c|c|c|c|c|c|c|c|c|}
\hline \multirow[t]{2}{*}{ Stage } & \multirow[b]{2}{*}{$\mathrm{n}=$} & \multicolumn{8}{|c|}{$\begin{array}{c}\text { Percent bivalents with } n \\
\text { nodules/bars or chiasmata }\end{array}$} \\
\hline & & $0 \quad 1$ & 2 & 3 & 4 & 5 & 6 & 7 & $7>7$ \\
\hline \multirow[t]{2}{*}{ Late zygotene } & $\mathrm{O}$ & 45 & 10 & 14 & 17 & 16 & 16 & & $5 \quad 12$ \\
\hline & $\mathrm{E}$ & 47 & 11 & 14 & 15 & 14 & 11 & & 815 \\
\hline \multirow[t]{2}{*}{ Pachytene 1} & $\mathrm{O}$ & 417 & 20 & 21 & 14 & 7 & 7 & & 45 \\
\hline & $\mathrm{E}$ & 715 & 19 & 17 & 14 & 10 & 7 & & 46 \\
\hline \multirow[t]{2}{*}{ Pachytene 2} & $\mathrm{O}$ & 116 & 22 & 22 & 16 & 12 & 7 & & 33 \\
\hline & $\mathrm{E}$ & 715 & 19 & 18 & 15 & 11 & 7 & & 45 \\
\hline \multirow[t]{2}{*}{ Pachytene $3+4$} & $\mathrm{O}$ & 132 & 39 & 18 & 8 & 1 & 1 & & $\begin{array}{ll}0 & 0\end{array}$ \\
\hline & $\mathrm{E}$ & 1626 & 24 & 16 & 9 & 5 & 2 & & 10 \\
\hline \multirow[t]{2}{*}{ Pachytene 5} & $\mathrm{O}$ & 1843 & 27 & 8 & 3 & 0 & 0 & & $\begin{array}{ll}0 & 0\end{array}$ \\
\hline & $E$ & 2832 & 22 & 11 & 5 & 2 & 1 & & $\begin{array}{ll}0 & 0\end{array}$ \\
\hline Diakinesisa & 0 & 011 & 56 & 26 & 6 & 1 & 0 & & $\begin{array}{ll}0 & 0\end{array}$ \\
\hline
\end{tabular}

Table III.

Distribution of recombination nodules, bars and chiasmata among the bivalent arms of human spermatocytes. $O$, observed frequencies. $E$, expected frequencies if nodules and bars are distributed randomly among the bivalents.

\begin{tabular}{|c|c|c|c|c|c|c|c|c|c|}
\hline \multirow{2}{*}{ Stage } & \multirow[b]{2}{*}{$\mathrm{n}=$} & \multicolumn{8}{|c|}{$\begin{array}{l}\text { Percent bivalent arms with n } \\
\text { nodules/bars or chiasmata }\end{array}$} \\
\hline & & $0 \quad 1$ & 2 & 3 & 4 & 5 & 6 & & $>7$ \\
\hline \multirow[t]{2}{*}{ Late zygotene } & 0 & 1520 & 020 & 20 & 11 & 7 & 5 & 2 & 0 \\
\hline & $\mathrm{E}$ & 2019 & 920 & 16 & 11 & 7 & 4 & 2 & 0 \\
\hline \multirow[t]{2}{*}{ Pachytene 1} & $\mathrm{O}$ & 2431 & 122 & 12 & 6 & 3 & 1 & 1 & 1 \\
\hline & $E$ & 2727 & 720 & 13 & 7 & 4 & 2 & 1 & 0 \\
\hline \multirow[t]{2}{*}{ Pachytene 2} & $\mathrm{O}$ & 2130 & 026 & 14 & 6 & 1 & 0 & 0 & 0 \\
\hline & $\mathrm{E}$ & 2727 & 721 & 13 & 7 & 3 & 1 & 1 & 0 \\
\hline \multirow[t]{2}{*}{ Pachytene $3+4$} & 0 & 2554 & 418 & 4 & 1 & 0 & 0 & 0 & 0 \\
\hline & $\mathrm{E}$ & 4131 & 117 & 7 & 3 & 1 & 0 & 0 & 0 \\
\hline \multirow[t]{2}{*}{ Pachytene 5} & 0 & 4838 & 811 & 2 & 1 & 0 & 0 & 0 & 0 \\
\hline & $\mathrm{E}$ & $53 \quad 30$ & 012 & 4 & 1 & 0 & 0 & 0 & 0 \\
\hline Diakinesisa & 0 & 1064 & 424 & 2 & 0 & 0 & 0 & 0 & 0 \\
\hline
\end{tabular}

percent of all bivalents was devoid of nodules and bars compared to 16 percent resulting from the computer simulation, while bivalents with one and two nodules/bars comprised 71 percent of all bivalents against 50 percent if nodules/bars were placed randomly.

By pachytene stage 5 , the observed frequencies approached the simulated random ones except for a deficit of observed bivalents without nodules and bars and somewhat larger frequencies of bivalents with one and two nodules/bars.

A similar comparison was performed for the individual bivalent arms in order to see whether or not the nodules and bars are distributed among the bivalent arms in the same way as among the bivalents. The frequencies are given in Table III and show that also with respect to the arms are nodules and bars distributed at random at late zygotene and pachytene stages 1 and 2 while at stage $3+4,72$ percent of the arms possess one or two nodules/bars compared to 48 percent resulting from the random distribution. Thereafter, at stage 5 the observed and the computer generated frequencies again appear similar implying a random distribution.

Finally, the distribution of nodules and bars among the two arms of bivalents with 2, 3 and 4 nodules/bars was analyzed by comparing the observed frequencies of bivalents with the different possible combinations of nodules/bars per arm and the values resulting from the simulated random positioning. The relative frequencies given in Table IV reveal a fairly good correspondance between the observed and the random frequencies except for stage $3+4$ and to a lesser extent for late zygotene and pachytene stage 2 where the bivalents with nodules/bars in both arms are more frequently observed than expected when nodules are placed at random.

Tables II, III and IV also include the distribution of chiasmata among the bivalents and the bivalent arms at diakinesis, the listed frequencies being derived from the data published by HuLTÉN (29). By comparing the nodule/bar and chiasma distributions, it is apparent that the closest resemblance is encountered at pachytene stage $3+4$ and the observation that the bars at pachytene stage $3+4$ constitute 80 percent of the total number of nodules/bars thus indicates that the morphological change from a nodule to a bar 
Table IV.

Distribution of nodules/ bars among the two arms of bivalents. The figures are the relative frequencies of bivalents with 2,3 and 4 nodules/bars where a) both nodules/bars are in the same arm $(0+2)$ or one in each arm $(1+1)$; b) all 3 nodules/bars in the same arm ( $(0+3)$ or a $1+2$ distribution; and c) all 4 nodules/bars in the same arm or a $1+3$ or $2+2$ distribution. $O$, observed distribution. $E$, expexted frequencies if the nodules and bars were distributed randomly between the bivalent arms.

\begin{tabular}{lcccccccc}
\hline Stage & & $(1+1)$ & $(0+2)$ & $(1+2)$ & $(0+3)$ & $(2+2)$ & $(1+3)$ & $(0+4)$ \\
\hline Late zygotene & $\mathrm{O}$ & 50 & 50 & 67 & 33 & 33 & 42 & 25 \\
& $\mathrm{E}$ & 33 & 67 & 56 & 44 & 27 & 43 & 30 \\
\hline Pachytene 1 & $\mathrm{O}$ & 44 & 56 & 68 & 32 & 34 & 46 & 20 \\
& $\mathrm{E}$ & 40 & 60 & 62 & 38 & 29 & 47 & 24 \\
\hline Pachytene 2 & $\mathrm{O}$ & 47 & 53 & 64 & 38 & 40 & 45 & 15 \\
& $\mathrm{E}$ & 37 & 63 & 60 & 40 & 27 & 46 & 27 \\
\hline Pachytene 3+4 & $\mathrm{O}$ & 70 & 30 & 89 & 11 & 47 & 43 & 10 \\
& $\mathrm{E}$ & 39 & 61 & 62 & 38 & 30 & 47 & 23 \\
\hline Pachytene 5 & $\mathrm{O}$ & 39 & 61 & 69 & 31 & - & - & - \\
& $\mathrm{E}$ & 40 & 60 & 63 & 37 & & & \\
\hline Diakinesisa & $\mathrm{O}$ & 82 & 18 & 98 & 2 & 59 & 41 & 0 \\
\hline
\end{tabular}

a) Data from Hultén (29).

Table V.

Distribution of recombination nodules and bars among bivalents and bivalent arms at pachytene stage 2, and the distribution of nodules/bars between the arms of bivalents with 2,3 and 4 nodules/bars (see legend to Table IV). $O$, observed distribution. E, expected frequencies if nodules and bars were distributed randomly among bivalents and bivalent arms.

\begin{tabular}{|c|c|c|c|c|c|c|c|c|c|c|c|}
\hline & \multirow[b]{2}{*}{$\mathrm{n}$} & \multirow[b]{2}{*}{$=$} & \multicolumn{9}{|c|}{ Percent bivalents with $\mathrm{n}$ nodules and bars } \\
\hline & & & 0 & 1 & 2 & 3 & 4 & 5 & 6 & 7 & $>7$ \\
\hline Recombination & $\mathrm{O}$ & & 9 & 21 & 27 & 16 & 12 & 9 & 5 & 1 & 1 \\
\hline nodules & $\mathrm{E}$ & & 11 & 21 & 22 & 18 & 12 & 8 & 4 & 2 & 1 \\
\hline \multirow[t]{4}{*}{ Bars } & $\mathrm{O}$ & & 49 & 36 & 12 & 4 & 0 & 0 & 0 & 0 & 0 \\
\hline & $\mathrm{E}$ & & 52 & 32 & 12 & 4 & 1 & 0 & 0 & 0 & 0 \\
\hline & & & \multicolumn{9}{|c|}{ Percent bivalent arms with $\mathrm{n}$ nodules and bars } \\
\hline & $\mathrm{n}$ & $=$ & 0 & 1 & 2 & 3 & 4 & 5 & 6 & 7 & $>7$ \\
\hline Recombination & $\mathrm{O}$ & & 30 & 36 & 20 & 5 & 1 & 0 & 0 & 0 & 0 \\
\hline nodules & $\mathrm{E}$ & & 34 & 29 & 19 & 10 & 5 & 2 & 1 & 0 & 0 \\
\hline \multirow[t]{3}{*}{ Bars } & $\mathrm{O}$ & & 69 & 26 & 4 & 1 & 0 & 0 & 0 & 0 & 0 \\
\hline & $\mathrm{E}$ & & 72 & 22 & 5 & 1 & 0 & 0 & 0 & 0 & 0 \\
\hline & & & $(1+1)$ & $(0+2)$ & & $(1+2)$ & $(0+3)$ & & $(2+2)$ & $(1+3)$ & $(0+4)$ \\
\hline \multirow{2}{*}{$\begin{array}{l}\text { Recombination } \\
\text { nodules }\end{array}$} & 0 & & 56 & 44 & & 56 & 44 & & 37 & 50 & 13 \\
\hline & $E$ & & 39 & 61 & & 62 & 38 & & 28 & 47 & 25 \\
\hline \multirow[t]{2}{*}{ Bars } & $\mathrm{O}$ & & 56 & 44 & & 70 & 30 & & - & - & - \\
\hline & E & & 42 & 58 & & 66 & 34 & & - & - & - \\
\hline
\end{tabular}


Table VI.

Distribution of recombination nodules and bars among bivalents and bivalent arms at pachytene stage $3+4$, and the distribution of nodules/bars between the arms of bivalents with 2,3 and 4 nodules/bars (see legend to Table IV). $O$, observed distribution. E, expected frequencies if nodules and bars were distributed randomly among bivalents and bivalent arms. T, expected distribution of nodules/ bars if nodules were distributed at random, while the bars are positioned as in the reconstructed nuclei.

\begin{tabular}{|c|c|c|c|c|c|c|c|c|c|c|c|}
\hline & \multirow[b]{2}{*}{$\mathbf{n}$} & \multirow[b]{2}{*}{$=$} & \multicolumn{9}{|c|}{ Percent bivalents with $\mathrm{n}$ nodules and bars } \\
\hline & & & 0 & 1 & 2 & 3 & 4 & 5 & 6 & 7 & $>7$ \\
\hline \multirow[t]{2}{*}{ Bars } & $\mathrm{O}$ & & 11 & 40 & 35 & 11 & 3 & 0 & 0 & 0 & 0 \\
\hline & $\mathrm{E}$ & & 24 & 31 & 23 & 13 & 6 & 2 & 1 & 0 & 0 \\
\hline \multirow{4}{*}{$\begin{array}{l}\text { Bars+ } \\
\text { recombination } \\
\text { nodules }\end{array}$} & 0 & & 1 & 32 & 39 & 18 & 8 & 1 & 1 & 0 & 0 \\
\hline & $\mathrm{T}$ & & 7 & 31 & 32 & 18 & 8 & 3 & 1 & 0 & 0 \\
\hline & & & \multicolumn{9}{|c|}{ Percent bivalent arms with $\mathrm{n}$ nodules and bars } \\
\hline & $\mathrm{n}$ & $=$ & 0 & 1 & 2 & 3 & 4 & 5 & 6 & 7 & $>7$ \\
\hline \multirow[t]{2}{*}{ Bars } & 0 & & 36 & 49 & 12 & 2 & 0 & 0 & 0 & 0 & 0 \\
\hline & $\mathrm{E}$ & & 49 & 31 & 14 & 5 & 1 & 0 & 0 & 0 & 0 \\
\hline \multirow{4}{*}{$\begin{array}{l}\text { Bars }+ \\
\text { recombination } \\
\text { nodules }\end{array}$} & & & & & & & & & & & \\
\hline & 0 & & 25 & 54 & 18 & 4 & 0 & 0 & 0 & 0 & 0 \\
\hline & $\mathrm{T}$ & & 31 & 44 & 18 & 5 & 1 & 0 & 0 & 0 & 0 \\
\hline & & & $(1+1)$ & $(0+2)$ & & $(1+2)$ & $(0+3)$ & & $(2+2)$ & $(1+3)$ & $(0+4)$ \\
\hline \multirow[t]{2}{*}{ Bars } & $\mathrm{O}$ & & 72 & 28 & & 84 & 16 & & 56 & 39 & 6 \\
\hline & $E$ & & 40 & 60 & & 63 & 37 & & 31 & 47 & 22 \\
\hline \multicolumn{12}{|l|}{ Barst } \\
\hline recombination & $\mathrm{O}$ & & 70 & 30 & & 89 & 11 & & 47 & 43 & 10 \\
\hline nodules & $\mathrm{T}$ & & 60 & 40 & & 79 & 21 & & 44 & 47 & 9 \\
\hline
\end{tabular}

signifies that crossing over has occurred or is in progress. As the total number of nodules/bars amounts to 44 and the mean number of chiasmata is reported to be $50(29,34)$ it is, however, evident that all crossovers of a nucleus are not morphologically identifiable at this stage but that crossing over also occurs at previous and following substages. This conclusion is further supported by the differences between the two distributions, namely that univalents are extremely rare at diakinesis while one percent of the bivalents lacks a nodule or a bar at stage $3+4$. Also, bivalent arms without nodules or bars, which comprise 14 percent of all arms (excluding the short arms of the acrocentric bivalents), are more frequent than arms of nonacrocentric bivalents without chiasmata ( 2 percent).

\subsection{Comparison between the distribution of nodules and bars}

According to the results presented in sections 3.1-3.4 it is apparent that a nodule represents a potential of crossing over while the bar is the morphological manifestation of the actual occurrence of crossing over. In the following, the distributions of nodules and bars are compared in order to investigate whether both nodules and bars are distributed randomly among the bivalents and the bivalent arms at stage 2 and whether nodules are distributed independently of the bars at stages $3+4$ and 5 .

In the analysis of nodules and bars at stage 2 the observed number of nodules was placed at random among the bivalents by the computer as described in section 3.4. and the obtained frequencies of bivalents and bivalent arms with 
Table VII.

Distribution of recombination nodules and bars among bivalents and bivalent arms at pachytene stage 5 , and the distribution of nodules/bars between the arms of bivalents with 2,3 and 4 nodules/bars (see legend to Table IV). $O$, observed distribution. E, expected frequencies if nodules and bars were distributed randomly among bivalents and bivalent arms. $T$, expected distribution of nodules/ bars if nodules were distributed at random, while the bars are positioned as in the reconstructed nuclei.

\begin{tabular}{|c|c|c|c|c|c|c|c|c|c|c|c|}
\hline & \multirow[b]{2}{*}{$\mathrm{n}$} & \multicolumn{10}{|c|}{ Percent bivalents with $\mathrm{n}$ nodules and bars } \\
\hline & & $=$ & 0 & 1 & 2 & 3 & 4 & 5 & 6 & 7 & $>7$ \\
\hline \multirow[t]{2}{*}{ Bars } & 0 & & 31 & 43 & 17 & 6 & 3 & 1 & 0 & 0 & 0 \\
\hline & $\mathbf{E}$ & & 37 & 34 & 18 & 7 & 3 & 1 & 0 & 0 & 0 \\
\hline \multirow{5}{*}{$\begin{array}{l}\text { Barst } \\
\text { recombination } \\
\text { nodules }\end{array}$} & & & & & & & & & & & \\
\hline & 0 & & 18 & 43 & 27 & 8 & 3 & 0 & 0 & 0 & 0 \\
\hline & $\mathrm{T}$ & & 23 & 39 & 23 & 9 & 4 & 2 & 0 & 0 & 0 \\
\hline & & & \multicolumn{9}{|c|}{ Percent bivalent arms with $\mathrm{n}$ nodules and bars } \\
\hline & $\mathbf{n}$ & $=$ & 0 & 1 & 2 & 3 & 4 & 5 & 6 & 7 & $>7$ \\
\hline \multirow[t]{2}{*}{ Bars } & $\mathrm{O}$ & & 58 & 32 & 7 & 2 & 0 & 0 & 0 & 0 & 0 \\
\hline & $\mathrm{E}$ & & 61 & 27 & 9 & 2 & 1 & 0 & 0 & 0 & 0 \\
\hline \multirow{4}{*}{$\begin{array}{l}\text { Barst } \\
\text { recombination } \\
\text { nodules }\end{array}$} & & & & & & & & & & & \\
\hline & 0 & & 48 & 38 & 11 & 2 & 1 & 0 & 0 & 0 & 0 \\
\hline & $T$ & & 51 & 33 & 12 & 3 & 1 & 0 & 0 & 0 & 0 \\
\hline & & & $(1+1)$ & $(0+2)$ & & $(1+2)$ & $(0+3)$ & & $(2+2)$ & $(1+3)$ & $(0+4)$ \\
\hline \multirow[t]{2}{*}{ Bars } & 0 & & 53 & 47 & & 67 & 33 & & - & - & - \\
\hline & $\mathbf{E}$ & & 40 & 60 & & 64 & 36 & & - & - & - \\
\hline \multirow{2}{*}{$\begin{array}{l}\text { Bars+ } \\
\text { recombination } \\
\text { nodules }\end{array}$} & 0 & & 39 & 61 & & 69 & 31 & & - & - & - \\
\hline & $T$ & & 43 & 57 & & 63 & 37 & & - & - & - \\
\hline
\end{tabular}

$0,1,2$, etc. nodules were compared to the observed values. As seen in Table $\mathrm{V}$, the observed and the computer generated frequencies were very similar as were the observed and random distributions of nodules between the two arms of individual bivalents. The computer was then asked to place, also at random, a number of bars equal to those observed and to determine the three sets of frequencies. As it can be seen from Table V, there is good agreement between the computer generated frequencies and the observed ones. This shows that the nodules which are transformed into bars at pachytene stage 2 are selected at random among all nodules of the nucleus.

If the observed distribution of bars alone at stage $3+4$ is compared to the distribution obtained by random placement of the same num- ber of bars (Table VI), marked differences are apparent as is also indicated by the combined nodule/bar distribution described in section 3.4. At this stage, bars are distributed evenly among the bivalents and the bivalent arms and in bivalents with 2,3 and 4 bars there is a strong preference for an even rather than a random distribution between the two arms of a bivalent.

In order to see if the 11 remaining nodules at stage $3+4$ are remnants of the population present at the previous stage and thus are distributed at random, or if their location is affected by the same factors which govern the selection of sites where bars are present, the following simulation experiment was carried out: The computer was supplied with the position of all bars in the 20 nuclei at stage $3+4$ and then asked to position at random the actual number of nodules found in 
each nucleus, the only restriction being that the positions occupied by bars were not available. As shown in Table VI the resultant distribution deviates markedly from that observed, the frequency of bivalents and bivalent arms without recombination structures being less than those generated by the computer simulation (Table VI). This implies that the nodules at stage $3+4$ are not located randomly but are to a large extent confined to bivalents and bivalent arms devoid of bars. Hence, placement of nodules at this stage reduces the number of bivalents and bivalent arms without nodules and bars. The data on the two groups of recombination structures may accordingly be pooled.

The results of a similar analysis performed for nuclei at stage 5 were less clear cut since only 9 nuclei were analyzed and some of the structures termed nodules may, in fact, represent remnants of bars rather than newly positioned recombination nodules. It is apparent, however, from the data shown in Table VII, that bars also at this stage are nonrandomly placed among bivalents and bivalent arms although not to the same extent as at stage $3+4$. It can also be seen from the data in Table VII that the nodules (mean of seven per nucleus) are not placed independently of the bars, but are preferentially located on bivalents and bivalent arms which are devoid of a bar. Hence, also at this stage may the data for bars and recombination nodules be pooled.

\subsection{Distribution of nodules and bars along the bivalents \\ 3.6.1. Classification of bivalents}

A prerequisite for the detailed analysis of the distribution of nodules and bars along the bivalents is an identification of the individual bivalents. Previous studies $(23,24,42)$ have shown that 14 of the autosomal bivalents i.e., numbers 1-5, 9, 12, 16-22 can be identified by their length and centromere index in combination with marker structures such as the secondary constriction on bivalents 1,9 and 16 and the nucleolus organizing regions on bivalents 13,14 , 15,21 and 22 . Subsequently it was shown (5) that the bivalent which by length and centromere index is identified as number 6 possesses a mor- phological marker consisting of a sphere of low electron density fibers associated with the short arm. This marker is present from late zygotene to pachytene stage 7. During the course of this study, an additional marker with a fine structure similar to that of the marker on chromosome 6 was identified. It is associated with the short arm next to the centromere region of one of the shorter bivalents (Figure 5). The marker was present in all but two of the 20 nuclei at stage $3+4$, absent in nearly all nuclei at later stages and identifiable with some difficulty in nuclei at stage 2 . In 17 of the 20 nuclei at stage $3+4$, the bivalent was classified as number 17 on the basis of length and centromere index, while the bivalent was classified as number 8 in two nuclei and number 11 in one nucleus. This result confirms that length and centromere index alone in most cases are sufficient parameters for the identification of individual bivalents. In summary it may thus be concluded that the identification is ambiguous for bivalents $13,14,15$ and 8,10 and 11 , that bivalents $1,6,9$ and 17 always are correctly identified and that the remainder of the bivalents only in rare cases are classified incorrectly.

\subsubsection{The distribution of nodules and bars along the bivalents}

The distribution of nodules and bars along the autosomal bivalents at late zygotene and pachytene stages $1,3+4$ and 5 is shown in Figures 6 and 7 expressed as the number of nodules/ bars per $0.67 \mu \mathrm{m}$ segment per nucleus. As described previously (42), the number of nodules per segment at late zygotene is only determined for segments which are paired in five or more of the ten analyzed nuclei. Hence, the frequencies of nodules for the interstitial, yet unpaired segments of the longer bivalents are not available. Furthermore, the observations on the positions of nodules and bars are pooled as both strucures appear to be distributed in the same manner among the bivalents and the bivalent arms at all stages.

The following characteristics are apparent when comparing the distributions presented in Figures 6 and 7: (1) In bivalents where pairing is complete or nearly so, i.e., bivalent numbers 8 , 11 and 13-22, nodules are present in high fre- 


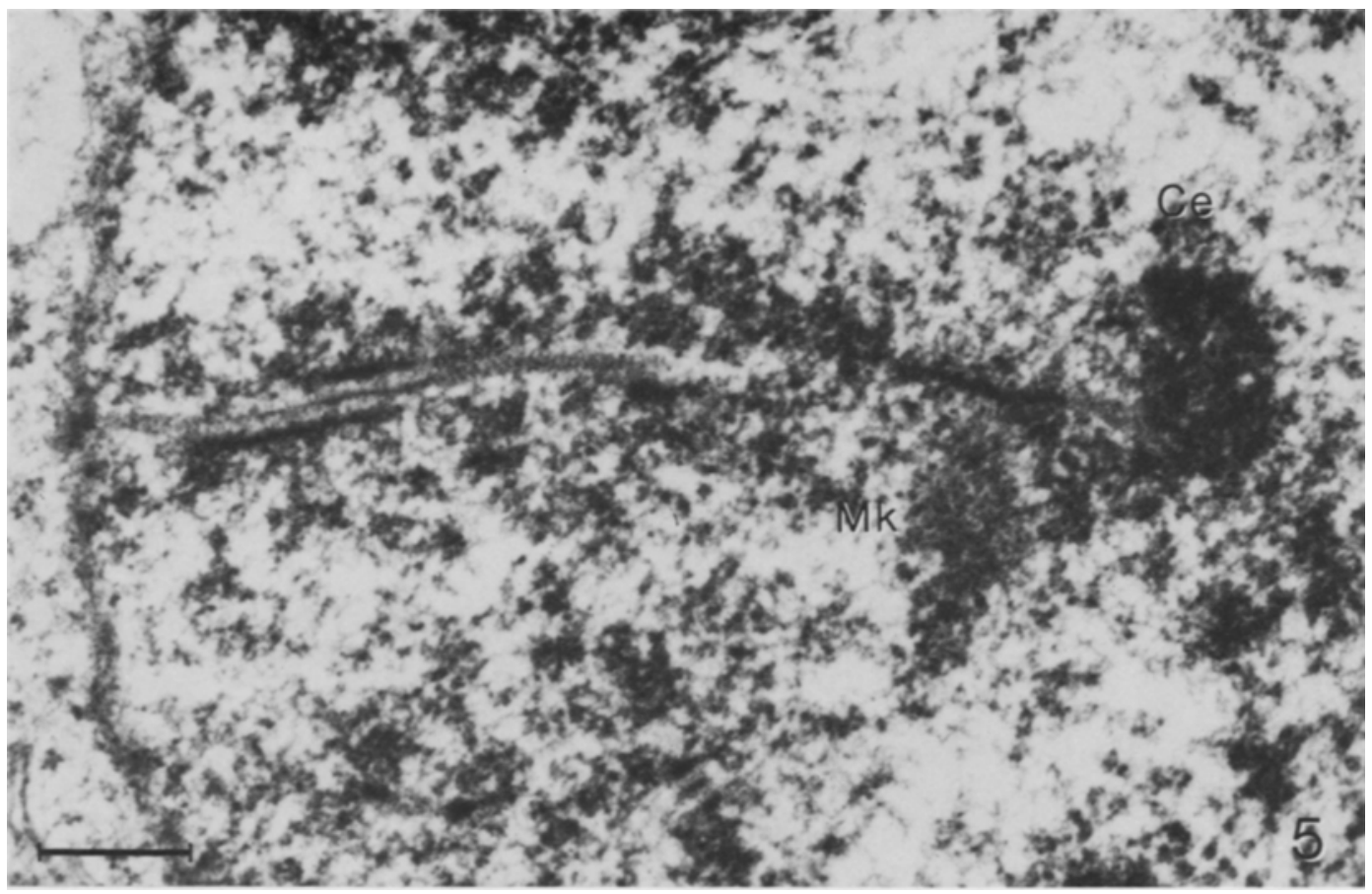

Figure 5. Electron micrograph of the synaptonemal complex of the short arm of bivalent 17 showing "marker 17" (Mk) next to the centromere region $(\mathrm{Ce})$. (Bar $=0.5 \mu \mathrm{m})$.

quency in the telomere regions while the frequency of nodules is low in the vicinity of the centromere region. Interstitially, one or more peaks are present. This is also the case at stages 1 and $3+4$ and generally the positions of the peaks and troughs coincide in the three distributions although some bivalents such as 14,15 and 17 , contain an additional peak at late zygotene. (2) A comparison of the distribution at late zygotene and pachytene stage 1 reveals that quantitative differences are most abundant in the telomere regions, while interstitially and around the centromere the nodule frequencies are more similar. This shows that the reduction in the number of nodules from 101 at late zygotene to 75 at pachytene stage 1 mainly affects distal segments. (3) The pachytene stage 1 distribution is very similar to that at stage $3+4$ in all shorter bivalents. In longer bivalents, i.e., numbers 1-5, 7,9 and 11 , there is a large excess of nodules interstitially at pachytene stage 1 . Hence, the nodule/bar distribution at stage $3+4$ may main- ly originate from that at stage 1 by a selective removal of nodules from interstitial segments. (4) The nodule/bar distributions at stages $3+4$ and 5 appear very similar (Figure 7), except in bivalents 2-5, 7-9 and 11 and the short arm of bivalent 1 , where nodules/bars are present at somewhat higher frequency in interstitial segments at stage 5 .

These comparisons indicate that in the longer chromosome arms specific segments with a higher affinity for recombination nodules and bars alternate with segments having a lower affinity. The mean distribution of nodules and bars at pachytene stages $1,3+4$ and 5 can be derived from that at late zygotene primarily by a differential removal of nodules. Removal of nodules cannot, however, explain the marked reduction in the number of bivalents without nodules or bars during pachytene stage 2 and the increase in interstitially positioned nodules at stage 5 relative to stage $3+4$. These changes require that additional nodules attach during this period. 
P.B. Holm \& S.W. RASMUSSEN: Human meiosis VI
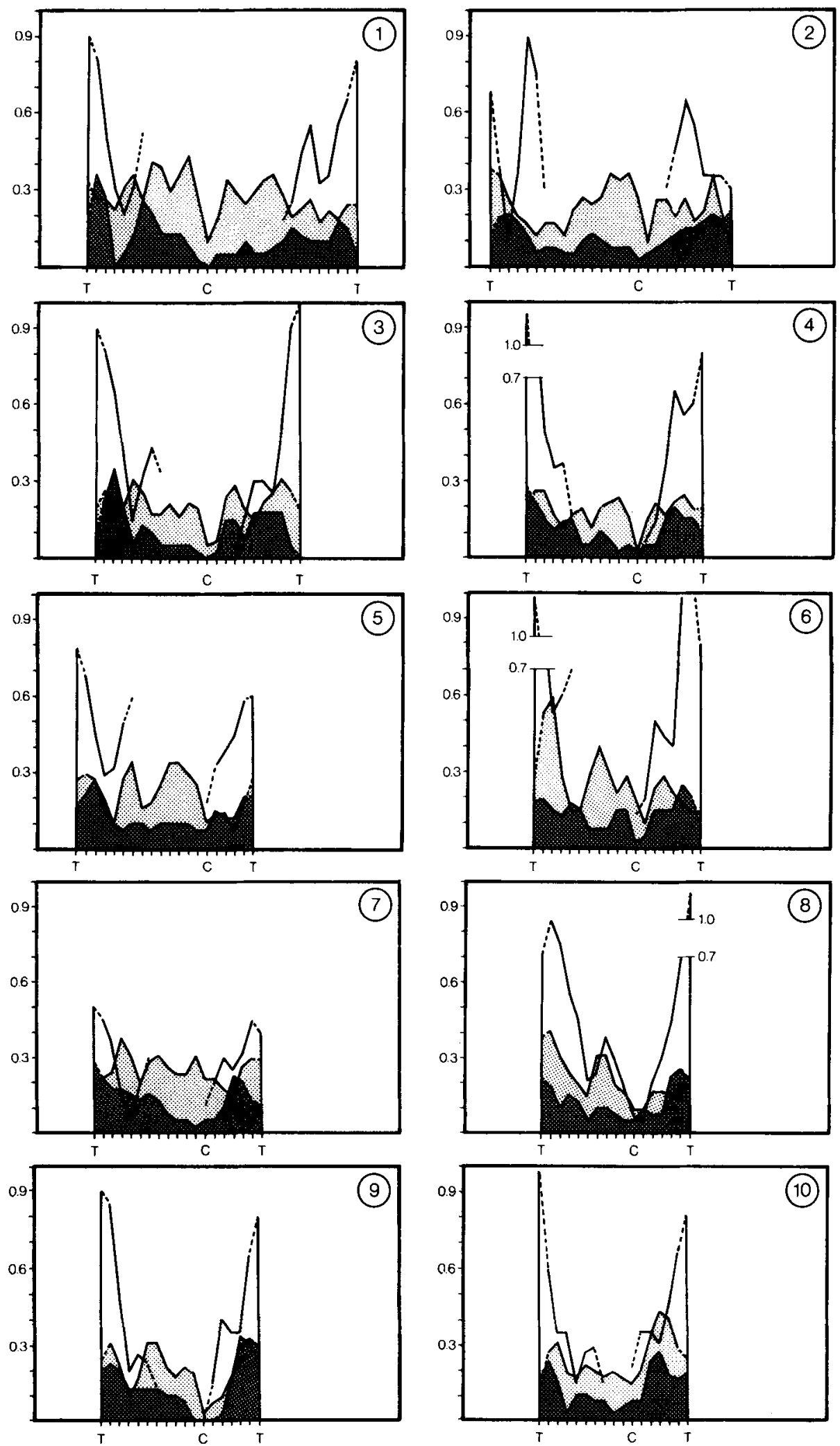

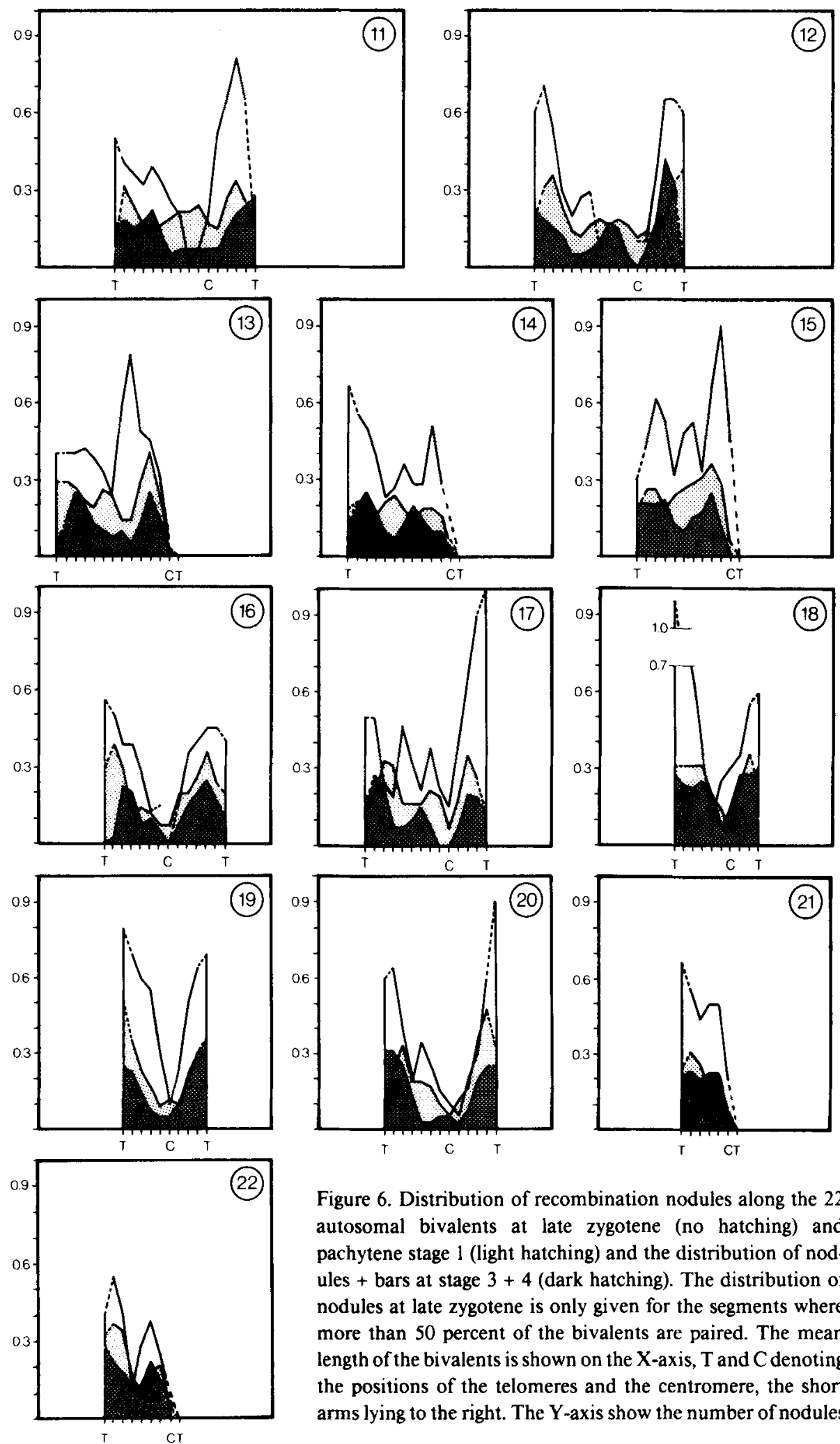

Figure 6. Distribution of recombination nodules along the 22 autosomal bivalents at late zygotene (no hatching) and pachytene stage 1 (light hatching) and the distribution of nodules + bars at stage $3+4$ (dark hatching). The distribution of nodules at late zygotene is only given for the segments where more than 50 percent of the bivalents are paired. The mean length of the bivalents is shown on the $X$-axis, $T$ and $C$ denoting the positions of the telomeres and the centromere, the short arms lying to the right. The $\mathrm{Y}$-axis show the number of nodules 
4 per nucleus per segment. The frequencies plotted in the diagram are the mean of two adjacent intervals (each equal to $0.67 \mu \mathrm{m}$ ) except for the values at the telomeres and the border betwen paired and unpaired regions which are represented by their actual value.
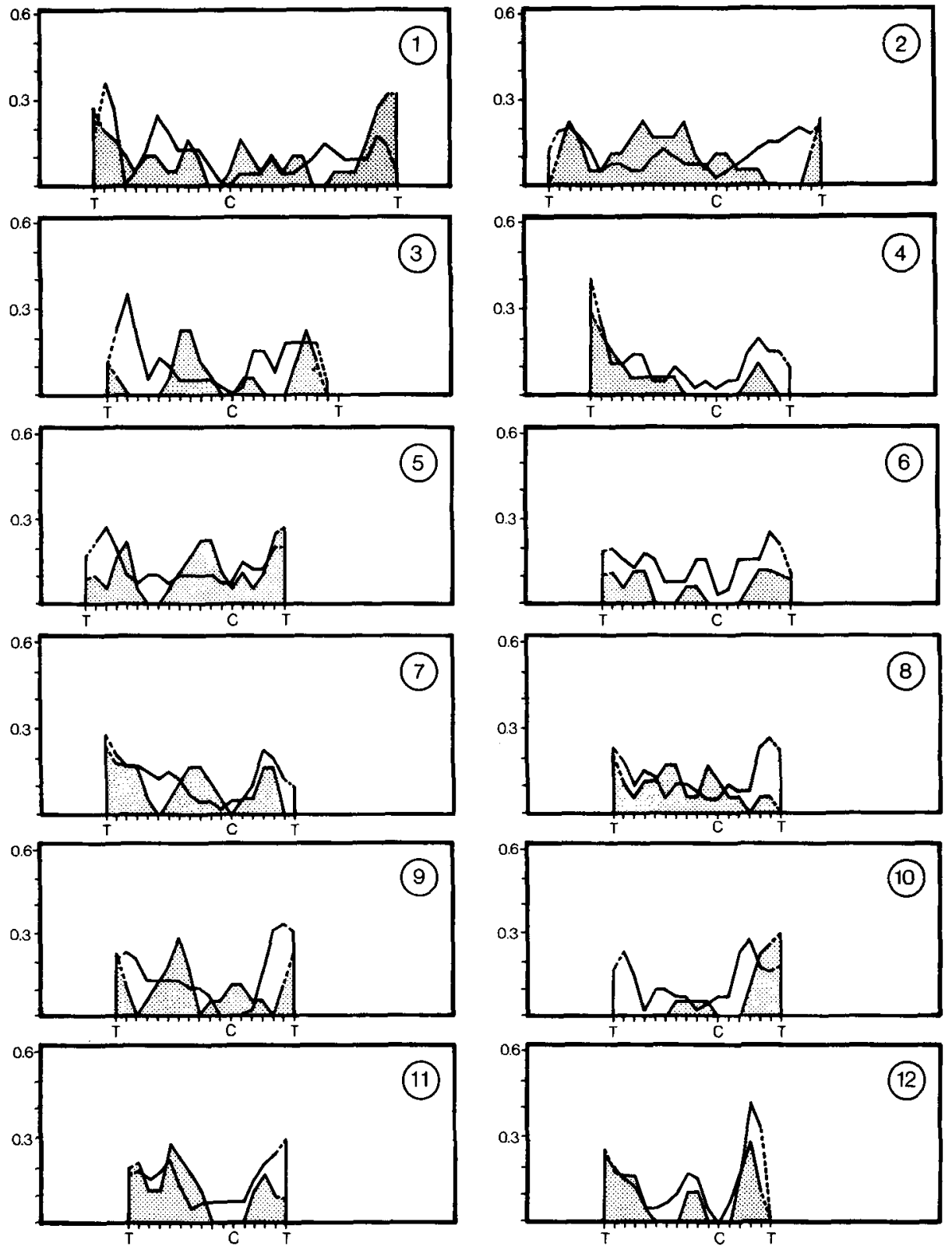

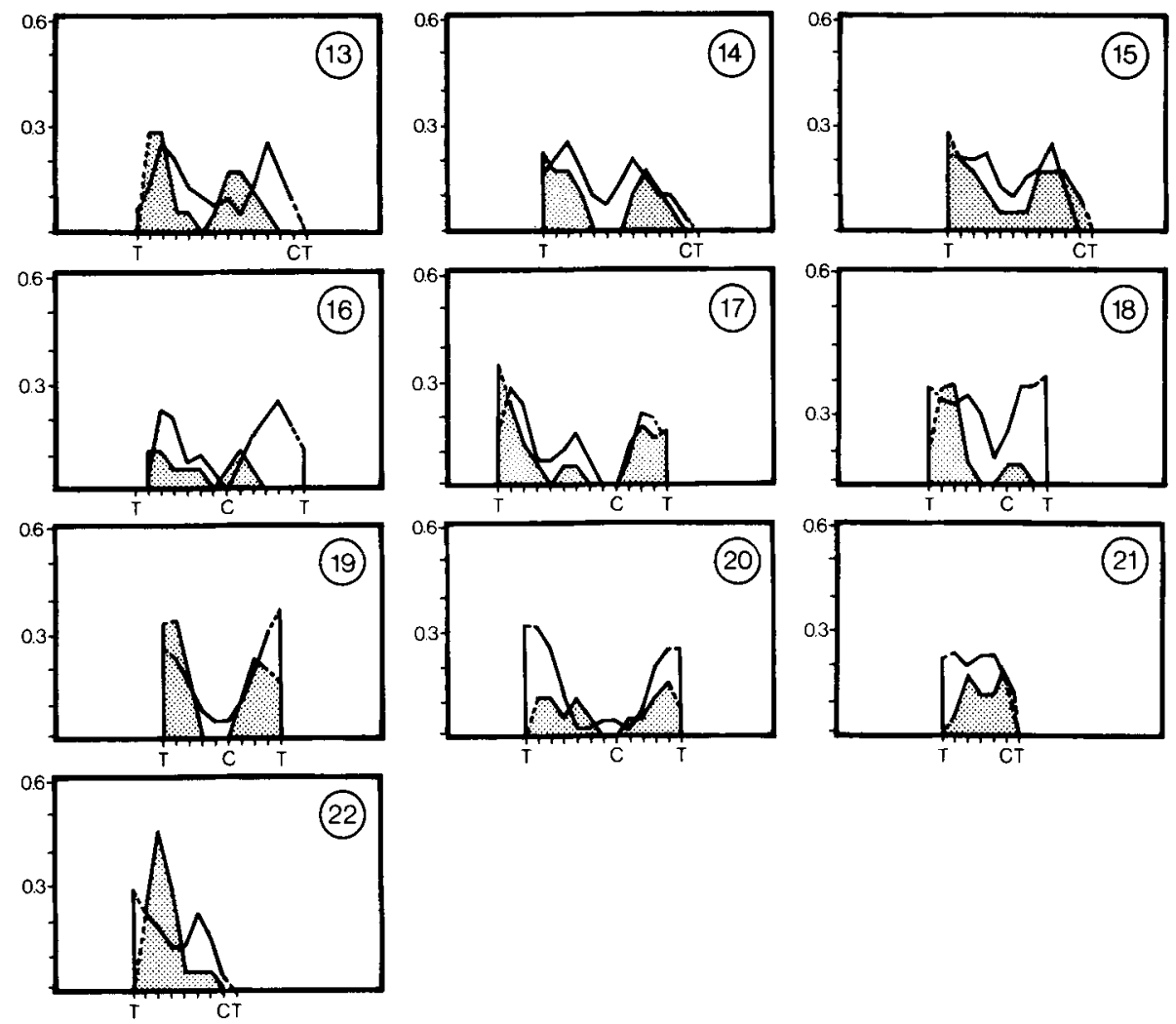

Figure 7. distribution of recombination nodules and bars along the 22 autosomal bivalents at stage $3+4$ (no hatching) and stage 5 (light hatching) (see also legend to Figure 6). 
P.B. Holm \& S.W. RasmusSEN: Human meiosis VI
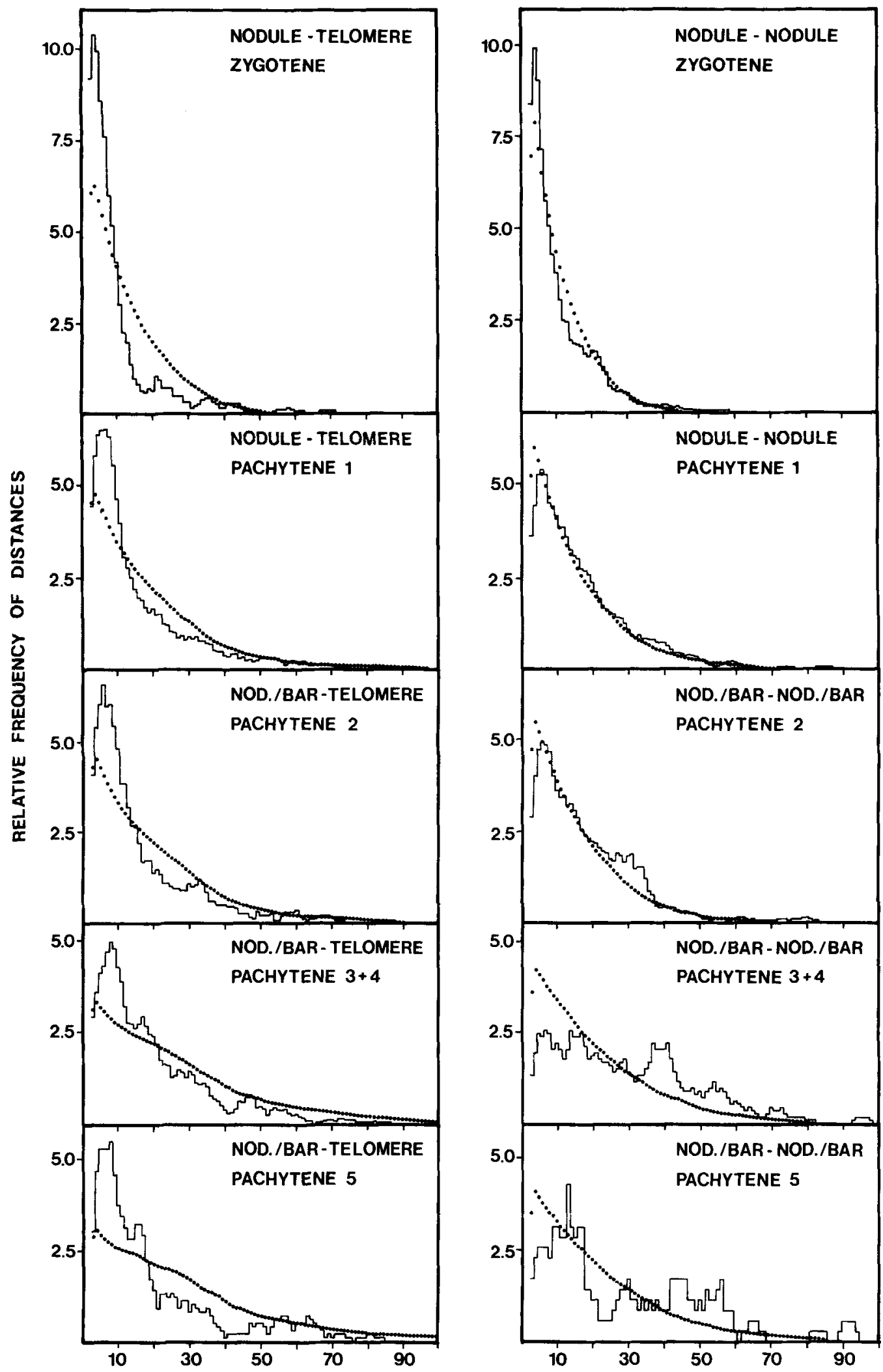
Figure 8. The distribution of the distances between the telomere and the first nodule or bar (left column) and the distance distribution for neighbour nodule/bars (right column) at late zygotene and pachytene stages 1 , $2,3+4$ and 5 . The histograms show the distribution of the observed distances expressed as relative frequencies of distances in $0.1 \mu \mathrm{m}$ classes. The values plotted in the diagrams are for each class the mean of that particular class and the two classes on either side. The dots represent the relative frequencies expected if nodules and bars were distributed at random among and along the bivalent arms.

\subsubsection{Distances from nodules/bars to telomeres and between neighbour nodules/bars}

The positions of nodules and bars relative to the telomere and to the nearest neighbour were analyzed by comparing the frequency of observed distances in $0.1 \mu \mathrm{m}$ intervals to the distances obtained by computer simulated random positioning of the observed number of nodules and bars on the reconstructed bivalent complement. The comparisons presented in Figure 8 show that from late zygotene to pachytene stage 1 there is an excess of nodules in the distal $1 \mu \mathrm{m}$ of the bivalent which is matched by a lower frequency of nodules in the interval from one to four micrometers, At stages $3+4$ and 5 there is an excess of distances up to $2 \mu \mathrm{m}$ and a deficit of longer distances. Hence, the most distal nodule or bar is located closer to the telomere during late zygotene and the entire pachytene period than can be accounted for by random placement.

In order to investigate whether neighbour nodules/bars are separated by a given distance along the bivalent arms, neighbour nodule/bar distances were compared with the random distance distribution obtained by the computer simulation experiment. Distances spanning the centromere were not included. As described previously (42) nodules are during late zygotene distributed" over the entire complement as if their positioning is independent of one another, the only exceptions being a small excess of neighbour distances below $0.8 \mu \mathrm{m}$ and a deficit of distances between 1 and $2 \mu \mathrm{m}$ at late zygotene. At pachytene stage 1 , the two distributions are virtually identical and only a minor deficit of very short distances is observed. At these two stages the placement of nodules does not indicate a requirement for a certain minimum distance between neighbour nodules.

From pachytene stage 2 , the frequencies of observed neighbour distances differ increasingly from those resulting from a random placement of nodules and bars; at stage 2 observed distances ranging from 2.5 to $3.5 \mu \mathrm{m}$ are more frequent than expected while distances outside this interval appear to follow the random distribution except for a minor deficit of very short observed distances. This pattern is further pronounced at stages $3+4$ and 5 where there is no longer a resemblance between the observed and the computer generated frequencies. Neighbour nodule/ bar distances of $0.5,1.5,4$ and $5.5 \mu \mathrm{m}$ at stage $3+$ 4 , and distances of about $1.5,3,4.5$ and $5.5 \mu \mathrm{m}$ at stage 5 are somewhat more frequent than others.

\subsubsection{Distribution of crossovers along the bivalents}

The observations described in the preceding sections have suggested that the appearance of a bar signifies that crossing over has taken place or is about to occur but that the site of crossing over is only identifiable by this structure during a limited period of time as some bars already become diffuse at stage 4 . It is therefore, not possible at any given stage to analyze the total distribution of crossovers in a single nucleus. The final crossover distribution can, however, be obtained by combining the distributions of bars at stage 2 and of nodules and bars at stages 3-7. Figure 9 illustrates the contribution of each stage, presented as a series of cumulative frequencies obtained by adding to the previous stage(s) the frequency of nodules/bars per segment for the next stage. The data for stages 6 and 7 are pooled due to the low number of nodules/bars present. The following conclusions may be drawn when comparing the contributions of each stage. (1) The distribution of bars at stage 2 is similar to the total distribution. (2) The contributions from stages $3,4,5$ and $6+7$ successively enhance the peaks and troughs along the bivalents. At stage 5 the interstitial peaks are prominently increased in some of the bivalents and also at stage $6+7$ changes in the distribution occur primarily at interstitial segments as seen in bivalents $1-5,8$, 11-14 and 17. 
P.B. HoLm \& S.W. RaSMUSSEN: Human meiosis VI
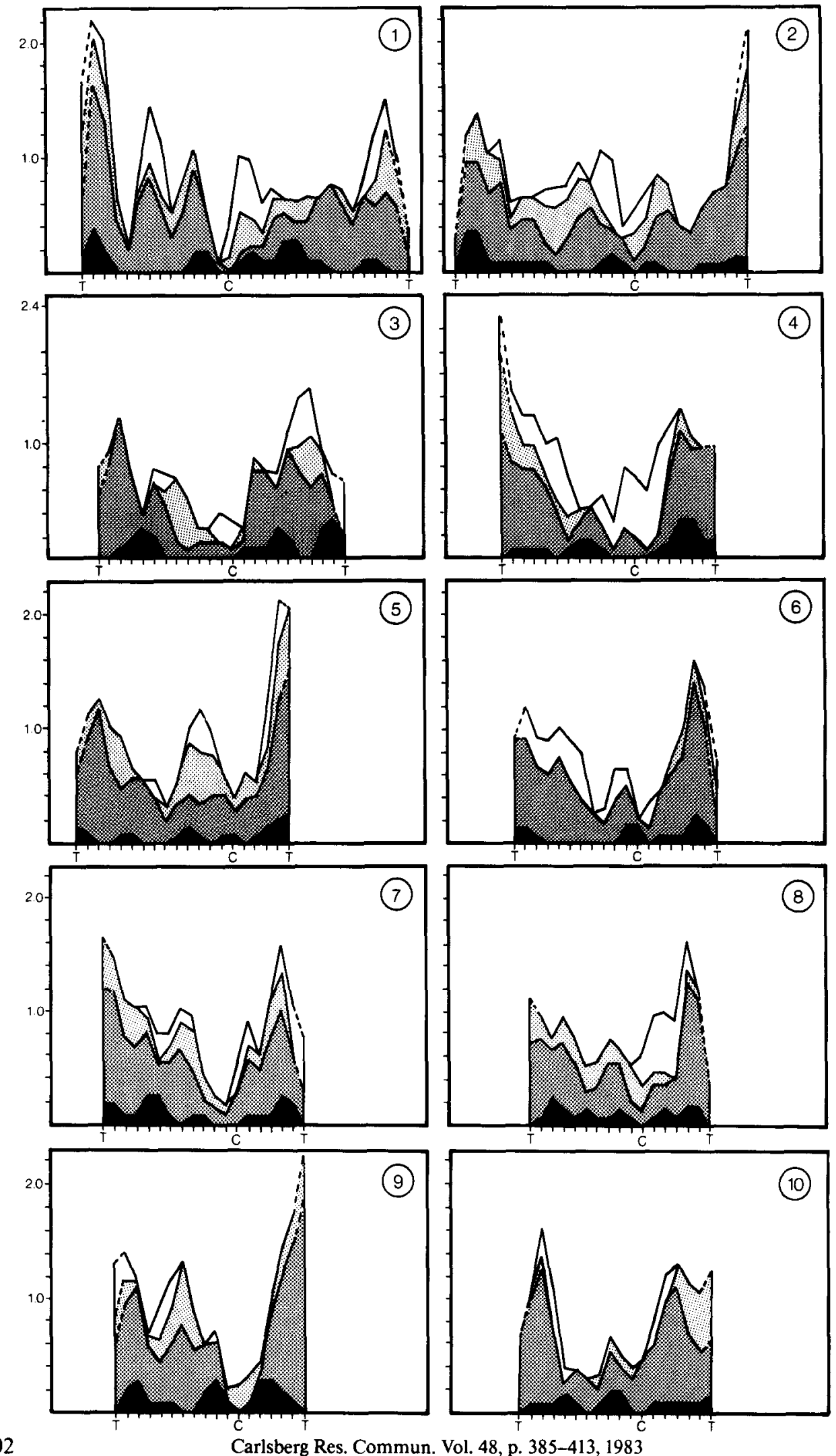


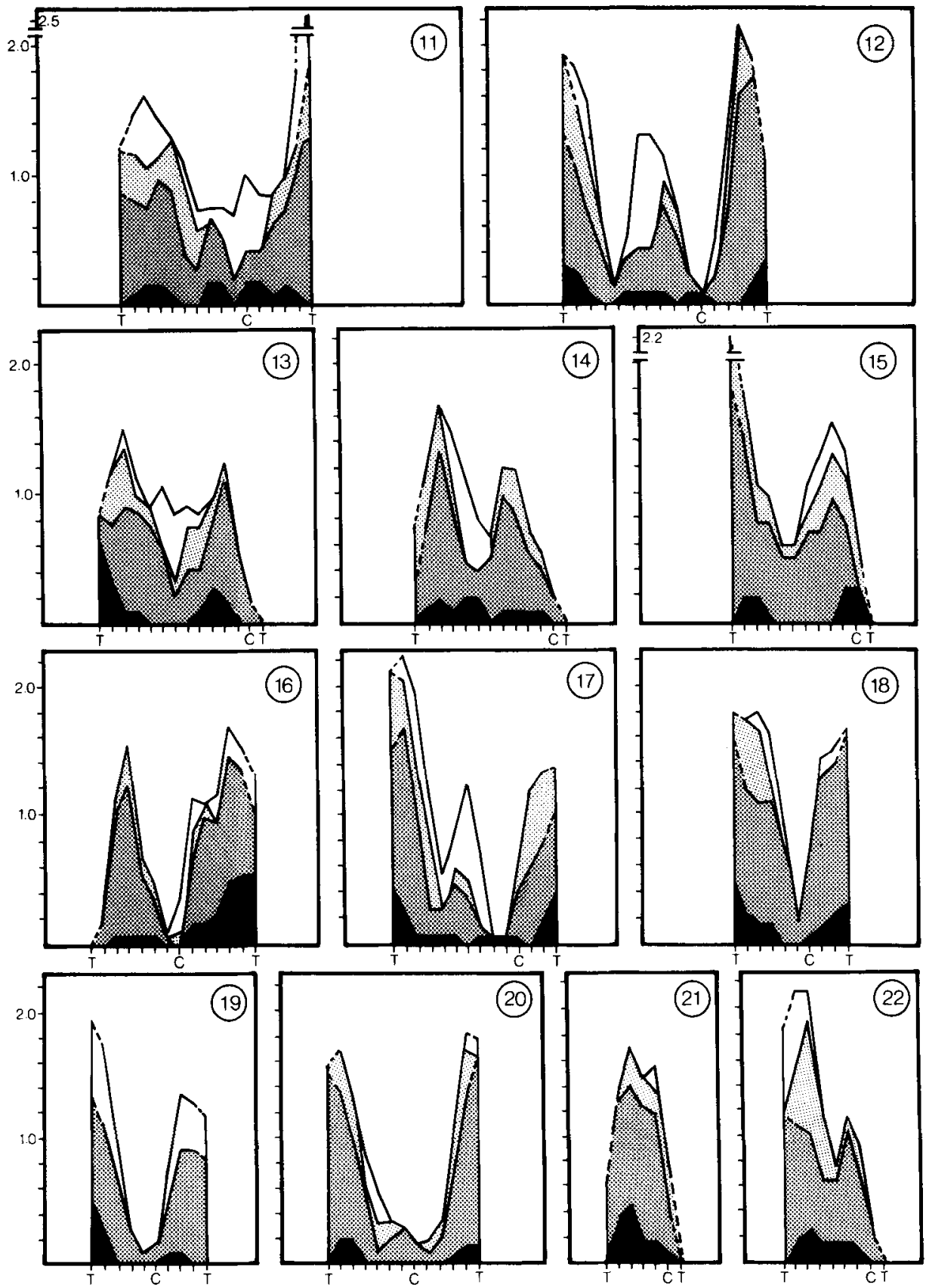

Figure 9. Cumulative distribution along the 22 autosomal bivalents of bars at stage 2 (black area) and nodules+bars at stages $3+4$ (dark hatching), stage 5 (light hatching) and stage $6+7$ (no hatching) (see also legend to Figure 6).

On the assumption that the time required for establishing a crossover, i.e., the attachment of a nodule, its transformation into a bar and its subsequent disappearance, is the same for all- crossovers irrespective of the time of occurrence or the position within the bivalent, the total accumulated distribution in Figure 9 represents the distribution of crossovers along the individual 
P.B. HOLM \& S.W. RASMUSSEN: Human meiosis VI
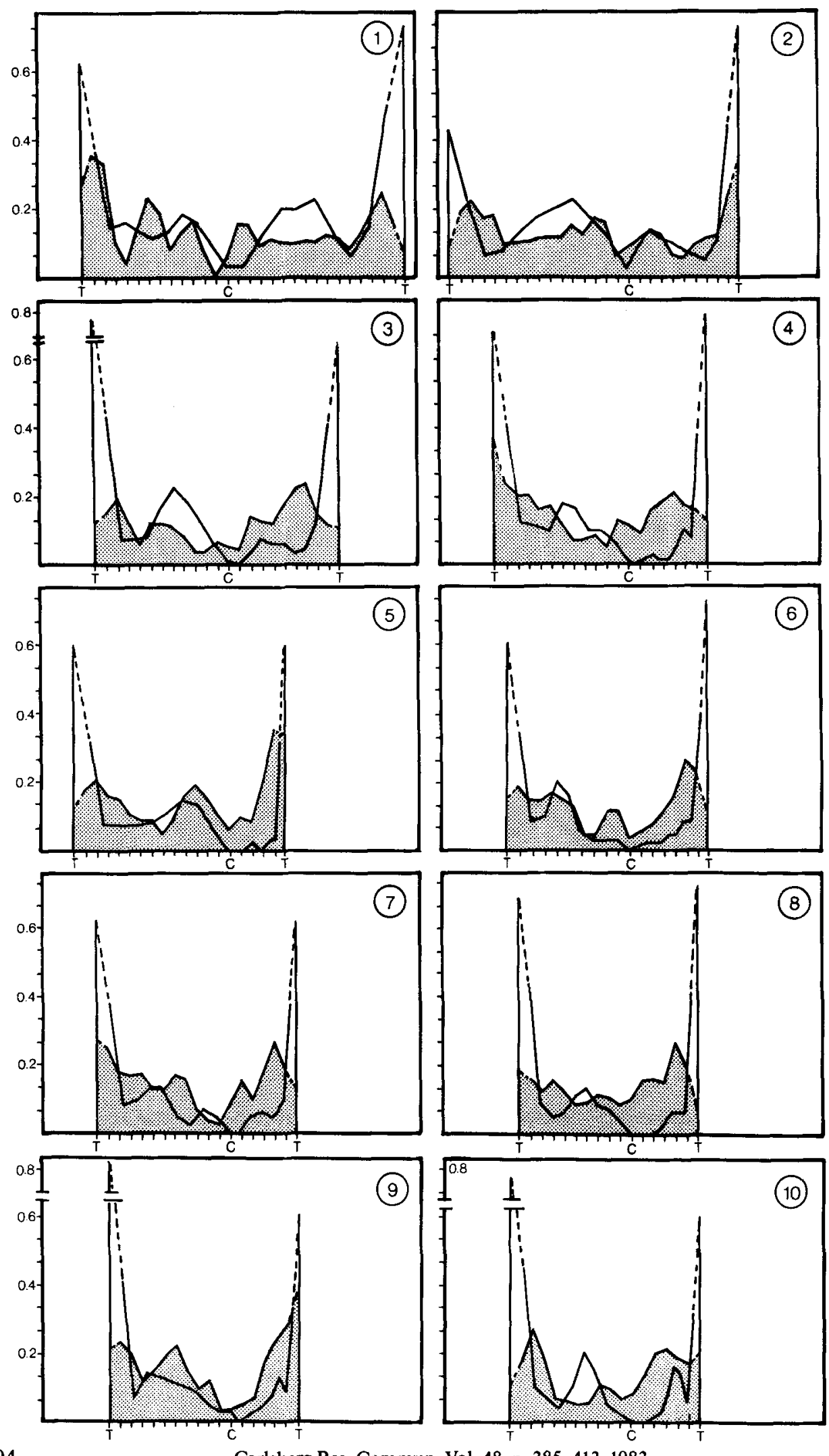


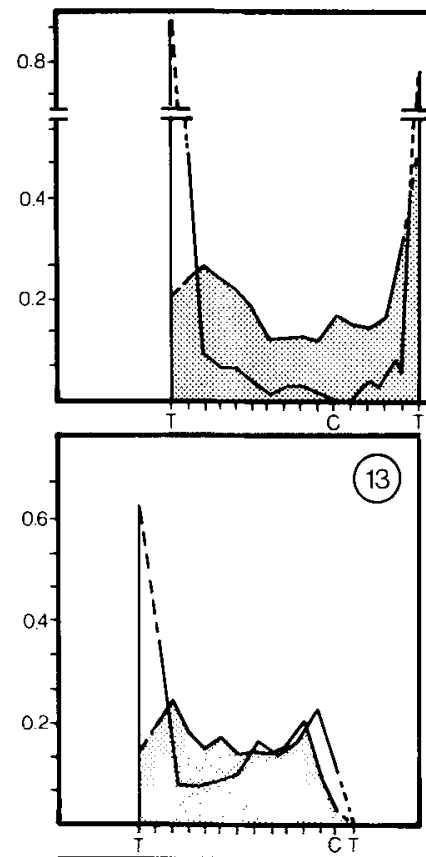

(11)
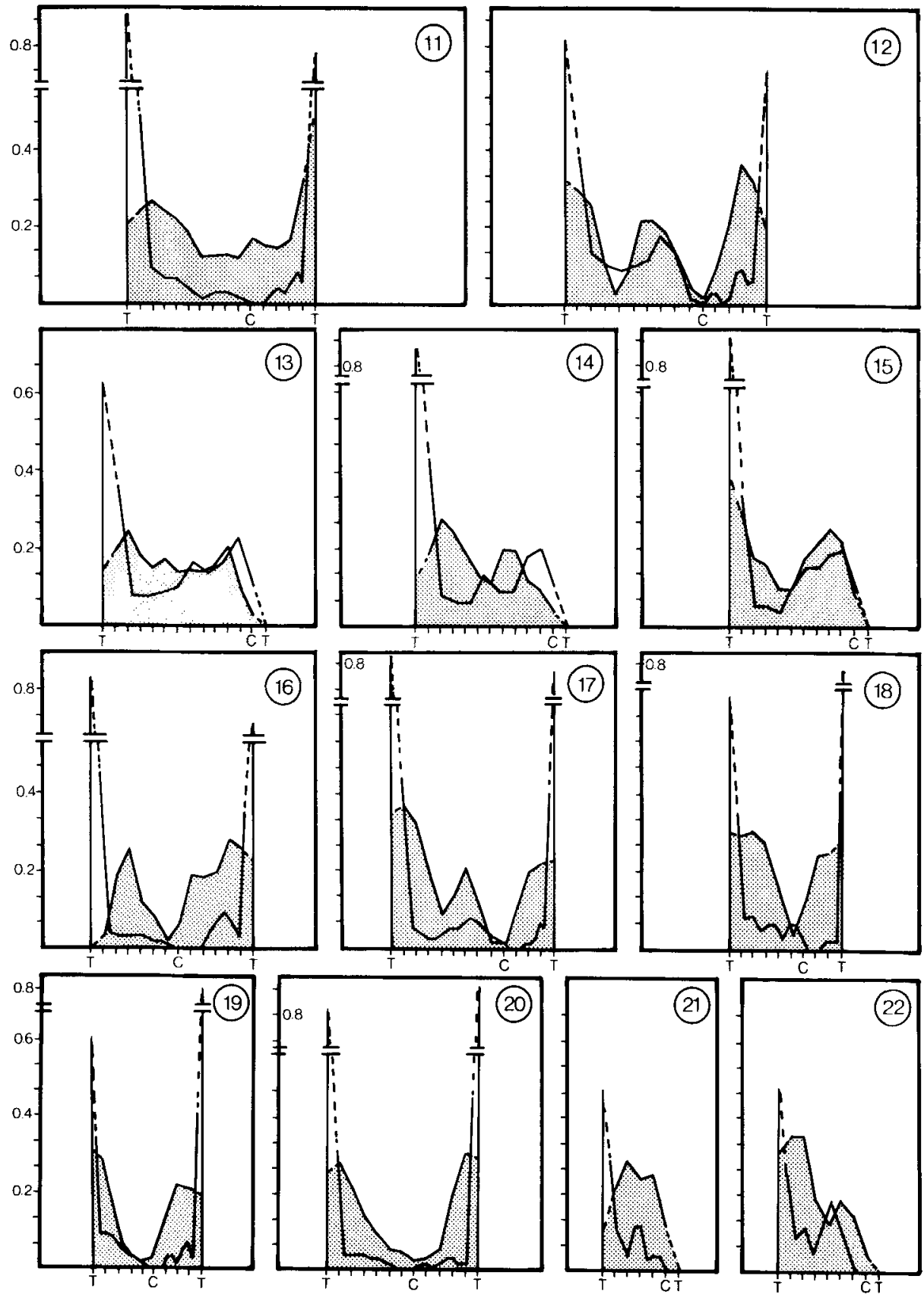

Figure 10. Distribution of crossovers (dark hatching) and distribution of chiasmata along the 22 autosomal bivalents. The distribution of chiasmata is compiled from the data by HULtÉN (29). The total frequency of crossovers has been corrected to match that of chiasmata, i.e., a mean of 50 per nucleus. As for the crossovers the chiasma frequencies plotted are the mean of two adjacent intervals. (See also the legend to Figure 6).

bivalents. The cumulative distributions show that the frequency of crossing over varies in a characteristic way along the individual bivalent arms and that the pattern is related to the length of the arms. The short arms of bivalents 3-12 and 16-20 all contain a single peak ranging in posi- 
Table VIII.

The estimated total number of crossovers ( $\mathrm{CO}$ ) per bivalent and the number of chiasmata at diakinesis. The length of the bivalents and arms is given in $\mu \mathrm{m}$.

\begin{tabular}{|c|c|c|c|c|c|c|c|}
\hline \multirow{2}{*}{$\begin{array}{l}\text { Bivalent } \\
\text { number }\end{array}$} & \multicolumn{2}{|c|}{ Short arm } & \multicolumn{2}{|c|}{ Long arm } & \multicolumn{2}{|c|}{ Total } & \multirow{2}{*}{$\begin{array}{l}\text { Number of } \\
\text { chiasmatac }\end{array}$} \\
\hline & $\mathrm{COb}$ & Length & $\mathrm{COb}$ & Length & $\mathrm{COb}$ & Length & \\
\hline 1 & 2.9 & 8.9 & 2.2 & 10.7 & 5.1 & 19.5 & 3.9 \\
\hline 2 & 1.8 & 7.0 & 3.0 & 10.6 & 4.8 & 17.5 & 3.6 \\
\hline 3 & 1.9 & 6.6 & 1.7 & 8.0 & 3.7 & 14.7 & 2.9 \\
\hline 4 & 1.5 & 4.7 & 2.5 & 8.3 & 3.9 & 12.9 & 2.8 \\
\hline 5 & 1.3 & 3.5 & 2.6 & 9.5 & 3.9 & 13.0 & 2.9 \\
\hline 6 & 1.3 & 4.9 & 1.9 & 7.4 & 3.2 & 12.3 & 2.7 \\
\hline 7 & 1.4 & 4.0 & 2.3 & 7.9 & 3.7 & 11.9 & 2.7 \\
\hline 8 & 1.4 & 3.8 & 1.7 & 6.8 & 3.1 & 10.5 & 2.6 \\
\hline 9 & 1.4 & 3.7 & 2.2 & 7.3 & 3.6 & 11.0 & 2.4 \\
\hline 10 & 1.4 & 4.1 & 1.6 & 7.1 & 3.1 & 11.1 & 2.5 \\
\hline 11 & 1.5 & 3.5 & 2.5 & 6.4 & 4.0 & 9.9 & 2.2 \\
\hline 12 & 1.5 & 3.0 & 2.3 & 7.6 & 3.8 & 10.5 & 2.7 \\
\hline 13 & - & - & 2.6 & 7.9 & 2.6 & 7.9 & 1.9 \\
\hline 14 & - & - & 2.4 & 7.3 & 2.4 & 7.3 & 1.9 \\
\hline 15 & - & - & 2.7 & 6.8 & 2.7 & 6.8 & 2.1 \\
\hline 16 & 1.7 & 3.9 & $0.9 \mathrm{a}$ & 4.6 & 2.6 & 7.5 & 2.2 \\
\hline 17 & $0.9 \mathrm{a}$ & 2.9 & 1.9 & 6.0 & 2.8 & 8.9 & 2.1 \\
\hline 18 & $1.0^{\mathrm{a}}$ & 1.7 & 1.6 & 4.2 & 2.7 & 5.8 & 1.9 \\
\hline 19 & $0.9 \mathrm{a}$ & 2.4 & $0.9 \mathrm{a}$ & 3.6 & 1.8 & 6.0 & 1.9 \\
\hline 20 & $1.0^{\mathrm{a}}$ & 3.6 & 1.3 & 4.5 & 2.3 & 8.2 & 1.9 \\
\hline 21 & - & - & 1.6 & 3.1 & 1.6 & 3.1 & 1.1 \\
\hline 22 & - & - & 2.1 & 4.5 & 2.1 & 4.5 & 1.2 \\
\hline Total number & & & & & 69.0 & 222.0 & 50.6 \\
\hline \multicolumn{7}{|c|}{$\begin{array}{l}\text { a) The arms used as standards for the conversion of the cumulative nodule/bar frequencies into crossover frequen } \\
\text { cies (section 3.6.5.) }\end{array}$} & $\begin{array}{l}\text { b) The frequencies are calculated assuming that } 5 \text { percent of the arms used as standards lack a chiasma. } \\
\text { c) Data from HuLrén (29). }\end{array}$ \\
\hline
\end{tabular}

tion from terminal to medial, the shorter arms having a more distal peak. The peak in some arms (the short arms of bivalents 3,5 and 8 ) appear somewhat bimodal with a distinct shoulder on the proximal side of the peak. Bivalent arms of intermediate length either have two distinct peaks (long arms of bivalents 3, 5, 6, 10, 1215,17 and the short arm of bivalent 2 ) or one major terminal to subterminal peak with one or more shoulders in the middle of the arm (long arms of bivalents 4 and 11). The two arms of bivalent 1 and the long arm of bivalent 2 contain three or four peaks.

The number and size of the peaks are, however, not alone a function of the arm length as can be seen by the pronounced differences in the distribution of crossover frequencies in arms of equal length such as the long arm of bivalents 4,5 and 6-12. Also, the relative size of the peaks varies considerably among the bivalents with two peaks.

\subsubsection{The total number of crossovers per nucleus}

As all crossovers are not identifiable at the same time, the total number of crossovers cannot be determined directly but must be derived from the accumulated frequencies of nodules and bars at stages $2-7$. On the assumption that the short arms of bivalents $17-20$ and the long arms of bivalents 16 and 19 get one crossover, the cumulative frequency of nodules and bars in these arms can be used to determine the frequency 
corresponding to one crossover. The mean cumulative frequency of these arms is 2.07 nodules/bars (range from 1.96 to 2.25 ) and as the cumulative frequency for all bivalents is 151 , the total number of crossovers per nucleus amounts to 73. The data published by Hultén (29) show that on the average 5 percent of the arms used as standards are devoid of a chiasma (of 240 arms analyzed, 15 lacked a chiasma and 3 had 2 chiasmata). Taking this into account, 2.18 nodules and bars correspond to one chiasma and the estimated total number of crossovers amounts to 69 (Table VIII).

\section{DISCUSSION}

\subsection{Morphological differentiation of recombination nodules}

Recombination nodules in human spermatocytes undergo a series of morphological changes from their binding to the synaptonemal complex during zygotene to their disappearence at late pachytene. From zygotene to pachytene stage 1, the nodules increase in size and density and their fibrillar connections to the lateral com. ponents of the complex become more prominent giving the structure the shape of a " $T$ ". The $T$ shaped nodules are particularly frequent at pachytene stage 2 , where the connections between the lateral components are often as distinct as the remainder of the nodule. Eventually, the spherical part of the nodule can no longer be recognized and the nodule consists entirely of an electron dense fusiform bar lying across the synaptonemal complex. During pachytene stages 3 , 4 and 5 , the bars increase in electron density and become more distinctly spindle shaped, frequently lying at an oblique angle to the complex. From stage 4 onwards, the bars resolve into spheres of medium dense fibers which eventually can no longer be recognized as distinct structures.

As discussed in section 4.2., the morphological changes in the nodules coincide with a reduction in number and a change from an initially random distribution among the bivalents into a distribution which is similar or identical to the distribution of crossovers and/or chiasmata $(9,10$, $25,26,42)$. Thus the morphological differentiation of a nodule into a bar marks a crossing over event.
Morphologically identical bars have previously been implicated in crossing over at pachytene in rat spermatocytes (37) and human spermatocytes $(3,4,46)$ and are also present in human oocytes (6). Analogous structural changes in recombination nodules during pachytene have, however, also been described in Bombyx (25) and Coprinus (26) and may be inferred from observations in Drosophila (10) and Neurospora (19). In these organisms, the early recombination nodules are small while those present at pachytene are larger and more electron dense.

The elimination of recombination nodules and the formation of chiasmata are morphologically expressed in various ways among different organisms. In Bombyx (25) and Coprinus (26), nodules of the mid pachytene type transform into irregular derivatives - chromatin nodules - from which more elaborate chromatin bridges - chiasmata - develop, while in Drosophila $(9,10)$, the large spherical pachytene nodules disappear without morphologically identifiable remnants, as is also the case for the bars in human spermatocytes (28).

\subsection{The numerical changes of recombination nodules}

There are 101 nodules associated with the synaptonemal complex at late zygotene in human spermatocytes which is considerably more than the estimated 70 crossovers per nucleus (see section 3.6.5.). A similar excess of nodules at the zygotene-pachytene transition has also been found in Schizophyllum (8) Bombyx (25) and Coprinus (26). On the assumption that unpaired regions at late zygotene upon completion of pairing will have the same probability of receiving a nodule as those segments already paired, the expected number of nodules at completion of pairing exceeds that of crossovers by a factor of 2.1, $1.8,1.7$, and 1.5 in human spermatocytes, Bombyx spermatocytes, Schizophyllum meiocytes and Coprinus meiocytes respectively. These results have led to the proposal $(25,26,42)$ that recombination nodules at zygotene are only capable of mediating nonreciprocal recombination without exchange of flanking markers. The coincident numerical and structural changes of the recombination nodules during early pachytene 
thus signify that the nodules become capable of mediating crossing over. This proposal has also been put forward by CARPENTER (10) who suggested that nonreciprocal recombination in Drosophila is mediated by the small ellipsoidal nodules while crossing over is mediated by the large spherical nodules which appear at mid pachytene.

In agreement with this OLSON and ZIMMERMAN (39) reported that when meiotic yeast cells were reverted to a mitotic cycle at different stages of the meiotic prophase, gene conversion without flanking marker exchange preceeded crossing over. Furthermore, as the nodules at zygotene are distributed at random among the bivalents and frequently in clusters along the bivalents, positive interference between nonreciprocal recombination events is expected to be low or absent. This is consistent with genetic investigations showing the virtual absence of interference between gene conversions without flanking marker exchange $(22,30,38)$. Finally, the average ratio between nonreciprocal and reciprocal recombination events is about $2: 1(16,41)$ i.e. approximately the same as the ratio between the number of recombination nodules at the zygotenepachytene transition and the number of crossovers.

\subsection{Distribution of nodules and bars among the bivalents}

The present analysis has shown that the numerous nodules at late zygotene are distributed randomly among the bivalents, the number of nodules per bivalent being directly proportional to the length of the paired segments. Following the decrease in the number of nodules at the zygotene-pachytene transition their distribution among the bivalents changes and by pachytene stage $3+4$, virtually all bivalents have at least one nodule or bar and seldom more than three. Most bivalent arms have one or two nodules or bars and in bivalents with 2, 3 and 4 nodules or bars the number of recombination structures in the two arms is roughly proportional to the length of the arms. At stage 5 and possibly also at stages 6 and 7 , nodules and bars appear to be less evenly distributed among the bivalents and the bivalent arms, but also at stage 5 , bivalents and bivalent arms with one nodule or bar are more frequent than expected from the simulation of a random placement. As almost all bivalent arms at diakinesis contain at least one chiasma (29), the nodules and bars present at stage 5-7 must to some extent be confined to bivalent arms devoid of crossovers at the previous stages ( 14 percent of all nonacrocentric arms at stage $3+4$ ).

These results imply that nodules continue to bind to the bivalents during mid and late pachytene but that the occurrence of one or more crossovers in a bivalent arm reduces its affinity for additional nodules. Thereby the number of binding sites available for nodules in the bivalent complement gradually diminishes as pachytene progresses and additional crossovers become preferentially confined to bivalent arms devoid of a crossover. It is thus apparent that the frequency of crossing over is regulated at the level of the individual bivalent and bivalent arm, a hypothesis supported by the observation that individual bivalents may be entirely devoid of crossovers, either as a regular feature of meiosis as in chromosome 4 of Drosophila or by mutation as described for the plant Hypochoeris (40). It should also be noted that positive chiasma position interference is a direct consequence if recombination nodule positioning is regulated by decreasing the probability for placement of additional nodules in a bivalent arm following the first crossover (see section 4.4.).

Similar numerical and distributional changes of nodules between late zygotene and mid pachytene have been reported in Bombyx spermatocytes (25), Coprinus meiocytes (26) and Drosophila oocytes $(9,10)$ and appear to be a general feature of meiosis. In Coprinus (26) it was shown by computer simulation that a reduction of the probability of receiving a nodule to one fifth following the attachment of the first nodule to the bivalent arm could generate the observed distribution of nodules among bivalents, among the two arms of a bivalent as well as the neighbour nodule distances.

The biological function of this mechanism is to reduce both the number of bivalents without chiasmata and the number of bivalents with a large number of chiasmata. Achiasmatic bivalents will separate upon elimination of the synaptonemal complex after pachytene into two independently segregating univalents while too 
many chiasmata may constitute a mechanical impediment to a proper separation of the homologues at anaphase I. Both cases would lead to increased levels of nondisjunction.

\subsection{The distribution of crossovers along the bivalents}

As described in section 3.6.4. the total crossover distribution can be calculated by adding the frequencies of bars at pachytene stage 2 to those of nodules and bars at stage 3-7, assuming that all crossover events are morphologically identifiable for the same period of time. The resultant distribution exhibits for almost all bivalents in humans a high frequency of recombination structures near the telomere region as is the case in Drosophila $(9,10)$, Sordaria (50), Schizophyllum (8) and Coprinus (26) but not in Bombyx (25). An intermediate situation is found in Neurospora (19). The centromere region has a low frequency of nodules and bars which has also been described in the other organisms studied, possible exceptions being Sordaria, Neurospora and Bombyx where the centromere region cannot be identified in the electron micrographs. Interstitial peaks were present only in human spermatocytes and not in any of the other organisms analysed.

In human spermatocytes the distribution of crossovers varies in a characteristic way along the bivalent arms. Short bivalent arms contain a single peak, intermediate sized arms generally show a bimodal distribution with a prominent subterminal peak and a less prominent one in the middle of the arm or proximally, while three or more peaks are present in the longest bivalent arms. Hence, the distribution of crossovers appears to be related to the length of the arm and in the longer arms several distinct regions with a high frequency of crossing over can be mapped. This result is consistent with the observation that certain classes of neighbour nodule distances are much more frequent than expected from a random positioning.

The functional significance of this uneven distribution of crossing over along the bivalents is at present unclear. It is conceivable that well spaced chiasmata in longer bivalent arms may facilitate the coorientation and disjunction of the homologues at metaphase I. The presence of a chiasma in the vicinity of the centromere may furthermore be advantageous for the coorientation of the centromeres. In agreement with this DARLINGTON (14) observed that bivalents which normally possess more than one chiasma are delayed in their coorientation and congression into the metaphase plate when only a single distal chiasma happened to be formed in them.

\subsection{The total number of crossovers}

The mean total number of crossovers per nucleus when estimated from the cumulated nodule/bar frequencies amounts to 73 when the short arms of bivalents 17-20 and the long arms of bivalents 16 and 19 are used as standards for the calculation. When taking into account that 5 percent of these arms on the average lack a chiasma (29) and hence also a crossover the total number is reduced to 69 crossovers per nucleus. Both estimates are, however, considerably in excess of the 50 chiasmata reported by HuLtén (29) and LAURIE et al. (34) for diakinesis bivalents.

In a recent paper SOLARI (46) reported an average number of bars per pachytene nucleus of 46.2 and showed that bars were preferentially present near the telomeres and seldomly in pericentromeric regions in agreement with the distribution of chiasmata. As these observations appear to have been made on nuclei from different substages (no details concerning the stage of the analyzed nuclei were reported) the pooled data are difficult to relate to those obtained in the present study except that the total number of crossovers reported by SOLARI is probably underestimated as no precautions were taken to compensate for the fact that all crossovers are not observable simultaneously.

\subsection{The regulation of crossing over}

The frequency and distribution of crossing over are regulated in several different ways: (1) Gene mutations in Drosophila (precondition mutants) (2) and plants (desynaptic mutants) $(18,31,32)$ can reduce the number of crossovers throughout the genome and change their distribution among and along the chromosome arms. (2) Regulation at the level of individual bivalents or bivalent arms is revealed by the elimination of crossing over from a particular bivalent (40) and by the nonrandom placement 
of crossovers to ensure at least one per bivalent (25, 26, section 4.3. of this paper). (3) Regulation at the level of chromosome segments is evidenced by the specific patterns found for the individual chromosome arms of the human complement (Figure 9). (4) Positive interference observed in genetic linkage studies implies that the occurrence of one crossover reduces the probability of another crossover being formed nearby. (5) Gene mutations in Neurospora and Schizophyllum $(13,33)$ can alter the frequency of crossing over in specific small segments of the linkage map.

A precise quantitative relationship cannot yet be established between the the distances determined by crossover frequencies in linkage studies and those mappable as recombination structures along the chromosome.

Short bivalent arms of human spermatocytes contain one crossover, rarely two (section 3.6.5.). The longer arms contain several crossovers but these are preferentially placed in the middle of two or three domains along the synaptonemal complex. The domain is likely to function like a short arm, i.e. once a crossover has occurred in the domain, the probability for a second one to be realized in the same domain is decreased. This limitation to the placement of a single crossover in a short arm and in a domain of a long arm is a source for positive interference in linkage studies.

The temporal development of the final crossing over distribution during pachytene stages 3-7 as described in section 3.6.4. has shown that interstitial peaks of the crossover distribution in several bivalents appear at stage 5 and that stages 6 and 7 contribute crossovers preferentially to the interstitial domains. Terminal domains appear thus to have a higher initial probability for crossing over than interstitial ones.

The low frequency of recombination structures in the centromere region and the crossover distribution within the domains of the bivalent arms are likely inherent characteristics of the associated chromatin. Genetic experiments with Drosophila have shown that the centromere region is always virtually devoid of crossing over irrespective of whether it by rearrangement has been moved to a different location (1). This may also be the case for other segments as evidenced by the constant genetic length of rearranged segments in inversion homozygotes in Drosophila (48). The numerous examples of changes in the crossover frequency in rearrangement heterozygotes of Drosophila, on the other hand probably arise as a result of incomplete and unstable pairing around the site of the pairing partner exchange, more recombination nodules being available for crossing over elsewhere in the genome (35). A possible candidate for the DNA regulating the frequency of crossing over might be the families of intermediate repeated DNA, nicked and repaired during pachytene and shown to be of high evolutionary stability (15).

Synaptonemal complex elimination at early diplotene appear to be initiated at the same sites in different nuclei, the position of these sites corresponding to the regions with a low crossover frequency, i.e., the border between domains (28). It is thus apparent that the initial degradation of the synaptonemal complex reflects structural differences in the complex, regions of high affinity for recombination nodules at pachytene being more resistant to degradation than regions of low affinity.

The result presented above for human spermatocytes are at variance with the classical hypothesis that the distribution of crossovers along the bivalents is determined by the establishment of an initial crossover at about the same distance from the centromere (36) or the telomere $(17,20$, 47) (differential distance), and subsequent crossover always forming at a certain distance away from the previous one (interference distance). As shown in section 3.6.4. the crossovers formed at pachytene stage 2 are not confined to just one region but appear to be distributed in the same way as the final distribution.

\subsection{Crossing over and chiasmata}

As outlined in section 4.5. the number of crossovers has been estimated to be about 70 per nucleus compared to a mean of 50 chiasmata at diakinesis as determined in air dried preparations (29). A comparison between the cumulative crossover frequencies along the bivalent arms after adjustment to show how 50 crossovers would be distributed and the distribution of 50 chiasmata according to HuLTÉn (29) within the same intervals is presented in Figure 10. The 
positions of chiasmata at diakinesis differ significantly from the positions of crossovers at pachytene. In particular the distal chiasmata are located closer to the telomeres than the crossovers. This conclusion is in contrast to that of Hultén (29) and and LAURIE et al. (34) who argued that terminalization during diplotene and diakinesis is negligible. Terminalization of chiasmata during diakinesis in humans is also indicated, if retained synaptonemal complex segments are used as indicators for crossovers (28). Whether the reduction of 70 crossovers at the end of pachytene to 50 light microscopically traceable chiasmata at diakinesis is due to terminalization events, remains to be seen.

\section{ACKNOWLEDGEMENTS}

It is a pleasure to thank D. vON WETTSTEIN for his constant support during the course of this work and especially for his critical review of the manuscript. Expert technical assistance was provided by JEAN SAGE and the financial support by grant BIO-E-417 DK(G) from the Commision of the European Communities and a Niels Bohr scholarship from the Royal Danish Academy of Sciences and Letters to PreBen B. Holm.

\section{REFERENCES}

1. BAKER, N.K.: Crossing over in heterochromatin. Amer. Naturalist 92, 59-60 (1958)

2. Baker, B.S., A.T.C. Carpenter, M.S. EsPOSITO, R.E. Esposito \& L. SANdLER: The gentic control of meiosis. Ann. Rev. Genet. 10, 53134 (1976)

3. Berthelsen, J.G., M. Fogh \& N.E. SkakKEBÆK: Electron microscopical analysis of meiotic chromosomes from human spermatocytes during and after treatment with steroid hormones. Carlsberg Res. Commun. 45, 9-23 (1980)

4. Berthelsen, J.G. \& N.E. SKAKKebeK: Ultrastructure of meiotic chromosomes in boys undergoing chemotherapy for leukemia. Leukemia Res. in press

5. Berthelsen, J.G., P.B. Holm \& S.W. RASMUSSEN: Three ultrastrucural markers on pachytene bivalents of human spermatocytes. Carlsberg Res. Commun. 45, 25-28 (1980)

6. Bojko, M.: Human meiosis VIII. Chromosome pairing and formation of the synaptonemal complex in oocytes. Carlsberg Res. Commun. $48,457-483$ (1983)
7. Byers, B. \& L. Goetsch: Electron microscopic observations on the meiotic karyotype of diploid and tetraploid Saccharomyces cerevisiae. Proc. Nat. Acad. Sci. USA 72, 5056-5060 (1975)

8. Carmi, P., P.B. Holm, S.W. Rasmussen, J. Sage \& D. ZICKLER: The pachytene karyotype of Schizophyllum commune analyzed by three dimensional reconstructions of synaptonemal complexes. Carlsberg Res. Commun. 43, 117 132 (1978)

9. CARPENTER, A.T.C.: Electron microscopy of meiosis in Drosophila melanogaster II. The recombination nodule - a recombination associated structure at pachytene? Proc. Nat. Acad. Sci. USA 72, 3186-3189 (1975)

10. Carpenter, A.T.C.: Synaptonemal complex and recombination nodules in wild type Drosophila melanogaster females. Genetics 92, 511541 (1979)

11. Carpenter, A.T.C.: Synaptonemal complex and recombination nodules in recombination deficient mutants of Drosophila melanogaster. Chromosoma (Berl.) 75, 259-292 (1979)

12. CARPENTER, A.T.C.: EM autoradiographic evidence that DNA synthesis occurs at recombination nodules during meiosis in Drosophila melanogaster. Chromosoma (Berl.) 83, 59-80 (1981)

13. CATCheside D.G.: The genetics of recombination. In: Genetics Principles and Perspectives. K.R. Lewis \& B. John eds. Edward Arnold, London Vol. 2, 172pp (1977)

14. Darlington, C.D.: The analysis of chromosome movement I. Podophyllum versipelle. Cytologia 7, 242-247 (1936)

15. Friedman, B.E., R.A. Bouchard \& H. Stern: DNA sequences repaired at pachytene exhibit strong homology among distantly related higher plants. Chromosoma (Berl.) 87, 409-424 (1982)

16. Fogel, S., R. Mortimer, K. Lusnak \& F. TavARES: Meiotic gene conversion: A signal of the basic recombination event in yeast. Cold Spring Harbor Symp. Quant. Biol. 43, 1325 1341 (1979)

17. Fox, D.P.: The control of chiasma distribution in the locust, Schistocerca gregaria (Forskảl). Chromosoma (Berl.) 43, 289-328 (1973)

18. Golubovskaya, I.N.: Genetic control of meiosis. Int. Rev. Cytol. 58, 247-290 (1979)

19. Gillies, C.B.: The relationship between synaptonemal complexes, recombination nodules and crossing over in Neurospora crassa bivalents and translocation quadrivalents. Genetics $91,1-17$ (1979) 
20. Henderson, S.A.: Chiasma distribution at diplotene in a locust Schistocerca gregaria. Heredity $18,173-190(1963)$

21. НовоLтh, P.: Chromosome pairing in allohexaploid wheat var. Chinese Spring. Transformation of multivalents into bivalents, a mechanisms for exclusive bivalent formation. Carlsberg Res. Commun. 46, 129-173 (1981)

22. Holliday, R.: Recombination and meiosis. Phil. Trans. R. Soc. London B. 277, 359-370 (1977)

23. Holm, P.B. \& S.W. Rasmussen: Human meiosis I. The human pachytene karyotype analyzed by three dimensional reconstructions of the synaptonemal complex. Carlsberg Res. Commun. 42, 283-323 (1977)

24. Holm, P.B. \& S.W. Rasmussen: Human meiosis III. Electron microscopical analysis of chromosome pairing in an individual with a balanced translocation 46,XY, $t(5 p-; 22 p+)$. Carlsberg Res. Commun. 43, 329-350 (1978)

25. Holm, P.B. \& S.W. Rasmussen: Chromosome pairing, recombination nodules and chiasma formation in diploid Bombyx males. Carlsberg Res. Commun. 45, 483-548 (1980)

26. Holm, P.B., S.W. Rasmussen, D. ZickleR, B.C. Lu \& J. SAGE: Chromosome pairing, recombination nodules and chiasma formation in the basidiomycete Coprinus cinereus. Carlsberg Res. Commun. 46, 305-346 (1981)

27. Holm, P.B. \& S.W. Rasmussen: Human meiosis V. Substages of pachytene in human spermatogenesis. Carlsberg Res. Commun. 48, 351-383 (1983)

28. Holm, P.B. \& S.W. Rasmussen: Human meiosis VII. Chiasma formation in human spermatocytes. Carlsberg Res. Commun. 48, 415-456 (1983)

29. HultéN, M.: Chiasma distribution at diakinesis in the normal human male. Hereditas 76, 55-78 (1974)

30. Hurst, D.D., S. Fogel \& R.K. Mortimer: Conversion-associated recombination in yeast. Proc. Nat. Acad. Sci. USA 69, 101-105 (1972)

31. JoNEs, G.H.: The control of chiasma distribution in rye. Chromosoma (Berl.) 22, 69-90 (1967)

32. Jones, G.H.: Correlated components of chiasma variation and the control of chiasma distribution in rye. Heredity 32, 375-387 (1974)

33. Koltin, Y. \& J. Stamberg: Genetic control of recombination in Schizophyllum commune: Location of a gene controlling B factor recombination. Genetics 74, 55-62 (1973)
34. Laurie, D.A., M. Hultén \& G. H. Jones: Chiasma frequency and distribution in a sample of human males: chromosomes 1,2, and 9. Cytogenet. Cell Genet. 31, 153-166 (1981)

35. LuCHESI, J.C \& D.T SUzukI: The interchromosomal control of recombination. Ann. Rev. Genet. 2, 53-86 (1968)

36. MATHER, K.: Crossing over. Biol. Reviews. 13, 252-292 (1938)

37. MoEns, P.B.: Lateral element cross connections of the synaptonemal complex and their relationship to crossing over and chiasmata in rat spermatocytes. Canad. J. Genet. Cytol. 20, 567-579 (1978)

38. Mortimer, R.K. \& S. Fogel: Genetic interference and gene conversion. In: Mechanism of Recombination, R.F. Grell, ed., Plenum Press, New York \& London pp. 263-275 (1974)

39. Olson, L.N. \& F.K. ZimmermanN: Meiotic recombination and synaptonemal complexes in Saccharomyces cerevisae. Mol. Gen. Genet. $166,151-159$ (1978)

40. PARKer, J.S.: Chromosome specific control of chiasma formation. Chromosoma (Berl.) 49, 391-406 (1975)

41. Perkins, D.D.: Crossing over of flanking markers is less than 50-percent among meiotic convertants. Genetics 91, s94 (1979)

42. Rasmussen, S.W. \& P.B. Holm: Human meiosis II. Chromosome pairing and recombination nodules in human spermatocytes. Carlsberg Res. Commun. 43, 275-327 (1978)

43. Rasmussen, S.W. \& P.B. Holm: Human meiosis IV. The elimination of synatonemal complex fragments from metaphase I bivalents of human spermatocytes. Carlsberg Res. Commun. 43, 423-438 (1979)

44. Rasmussen, S.W. \& P.B. Holm: Chromosome pairing in autotetraploid Bombyx females. Mechanism for exclusive bivalent formation. Carlsberg Res. Commun. 44, 101-125 (1979)

45. Rasmussen, S.W., P.B. Holm, B.C. LU, D. ZiCkler \& J. SAGE: Synaptonemal complex formation and distribution of recombination nodules in pachytene trivalents of triploid Coprinus cinereus. Carlsberg Res. Commun. 46, 347-360 (1981)

46. SOLARI, A.J.: Synaptonemal complexes and associated structures in microspread human spermatocytes. Chromosoma (Berl.) 81, 1-23 (1981)

47. SOUTHERN, D.I.: Chiasma distribution in truxaline grasshoppers. Chromosoma (Berl.) 22, 164-191 (1967)

48. Sturtevant, A.H.: Known and probable in- 
verted sections of the autosomes of Drosophila melanogaster. Carnegie Inst. Wash. Publ. 421, 1-27 (1931)

49. WestergaARd, M. \& D. von Wettstein: Studies on the mechanism of crossing-over IV. The molecular organization of the synaptinemal complex in Neottiella (Cooke) Saccardo (As- comycetes). Compt. Rend. Trav. Lab. Carlsberg 37, 239-268 (1970)

50. ZiCKLER, D.: Development of the synaptonemal complex and the "recombination nodules" during meiotic prophase in the seven bivalents of the fungus Sordaria macrospora Auersw. Chromosoma (Berl.) 61, 289-316 (1977) 